\title{
A Facile Synthesis and Discovery of Highly Functionalized Tetrahydro- pyridines and Pyridines as Antimycobacterial Agents
}

\author{
Suresh Kumar Raju, ${ }^{a}$ Michael Rajesh Stephen, ${ }^{a}$ Perumal Subbu, ${ }^{* a}$ Banerjee DebJani, ${ }^{b}$ \\ Yogeeswari Perumal, ${ }^{b}$ and Sriram DHARMARAJAN ${ }^{b}$ \\ ${ }^{a}$ Department of Organic Chemistry, School of Chemistry, Madurai Kamaraj University; Madurai 625021, India: and \\ ${ }^{b}$ Medicinal Chemistry \& Antimycobacterial Research Laboratory, Pharmacy Group, Birla Institute of Technology \& \\ Science-Pilani, Hyderabad Campus, Jawahar Nagar, Hyderabad-500 078, Andhra Pradesh, India. \\ Received August 17, 2009; accepted January 28, 2010; published online February 3, 2010
}

The four-component reaction of ethyl-3-oxo-4-(arylsulfanyl)butanoate, substituted aromatic aldehydes and ammonium acetate afforded novel ethyl 4-hydroxy-2,6-diaryl-5-(arylsulfanyl)-1,2,5,6-tetrahydro-3-pyridinecarboxylates. These tetrahydro-pyridine esters upon dehydrogenation with dichlorodicyanobenzoquinone (DDQ) afforded highly functionalized pyridines in excellent yields. These novel heterocycles were screened for their in vitro activity against Mycobacterium tuberculosis $\mathrm{H37Rv}$ using agar dilution method. Among the compounds screened, ethyl 2,6-di(2-bromophenyl)-4-hydroxy-5-(phenylsulfanyl)-3-pyridinecarboxylate was found to be the most active with a minimum inhibitory concentration of $1.33 \mu_{\mathrm{M}}$ against Mycobacterium tuberculosis and is 5.74 and 38.17 times more potent than the first line anti-tuberculosis (TB) drugs, ethambutol and pyrazinamide respectively.

Key words pyridinecarboxylate; Mannich reaction; stereochemistry; Mycobacterium tuberculosis

Piperidones serve an important role as intermediates to substituted piperidines ${ }^{1-3)}$ and they are found to be a part of more complex biologically active compounds. ${ }^{4}$ Apart from possessing analgesic, ${ }^{5,6)}$ anti-inflammatory, ${ }^{5)}$ central nervous system (CNS) depressant, ${ }^{7,8)}$ local anesthetic, ${ }^{7,9)}$ anticancer, ${ }^{10)}$ and antimicrobial activities, ${ }^{11)}$ piperidones are valuable synthetic intermediates for the preparation of various alkaloids and pharmaceuticals. ${ }^{12-14)}$ Piperidones have also been used as key chiral intermediates in the preparation of a large number of natural and synthetic compounds with significant anticancer, ${ }^{15)}$ anti human immunodeficiency virus (HIV) ${ }^{16)}$ and glycosidase inhibition ${ }^{17)}$ activities. Tetrahydropyridine derivatives are useful against several metabolic disorders and human ailments and are involved in monoamine oxidase based mechanism in Parkinson's disease ${ }^{18,19)}$ and as inhibitors of farnesyl transferase ${ }^{20)}$ and dihydroorate dehydrogenase $^{21)}$ and also play key roles in many disease processes.

The pyridine motif is found in various therapeutic agents, including numerous antihistamines, ${ }^{22)}$ antiseptic, ${ }^{23)}$ antiarrythmic, ${ }^{24)}$ antirheumatic, ${ }^{25}$ and other pharmaceutical agents and natural products. They also play a pivotal role in catalyzing both biological and chemical reactions. ${ }^{26)}$ Highly functionalized pyridines, including aryl- and heteroaryl-substituted derivatives, are widespread in the pharmaceutical and agrochemical sectors. ${ }^{27,28)}$ Due to their $\pi$-stacking ability, some pyridines are used in supramolecular chemistry as well. ${ }^{29,30)}$ Hence the synthesis of polysubstituted piperidones and pyridines is a topic of current interest. ${ }^{31,32)}$ This prompted us to perform the synthesis of poly substituted piperidines and pyridines and probe their biological potential.

It is pertinent to note that tuberculosis (TB) remains one of the major causes of disability and death worldwide. The WHO Global TB report released in March 2009 ${ }^{33)}$ shows that in 2007 there were an estimated 1.4 million new cases of tuberculosis among HIV-infected people and 456000 deaths. In addition, the number of people contracting TB is on the rise, as their immune systems are impaired by immunosuppressive drugs or substance abuse or HIV/AIDS. The emergence of multi-drug resistant TB (MDR-TB), defined as resistance to at least isoniazid and rifampicin, and the extensively drug resistant (XDR-TB) strains also impedes the discovery and the development of new drugs. ${ }^{34,35)}$ In the last 50 years, only a few drugs have been approved by the Food and Drug Administration (FDA) for combating TB, which reflects the difficulties rampant in the discovery and clinical testing of new agents and the lack of pharmaceutical industry research in this area ${ }^{36)}$ Hence, the discovery of fast-acting effective newer drugs to cure TB is imperative. Consequently, the novel poly functionalized piperidines (viz. enol form of piperidones) and pyridines synthesized in the present work

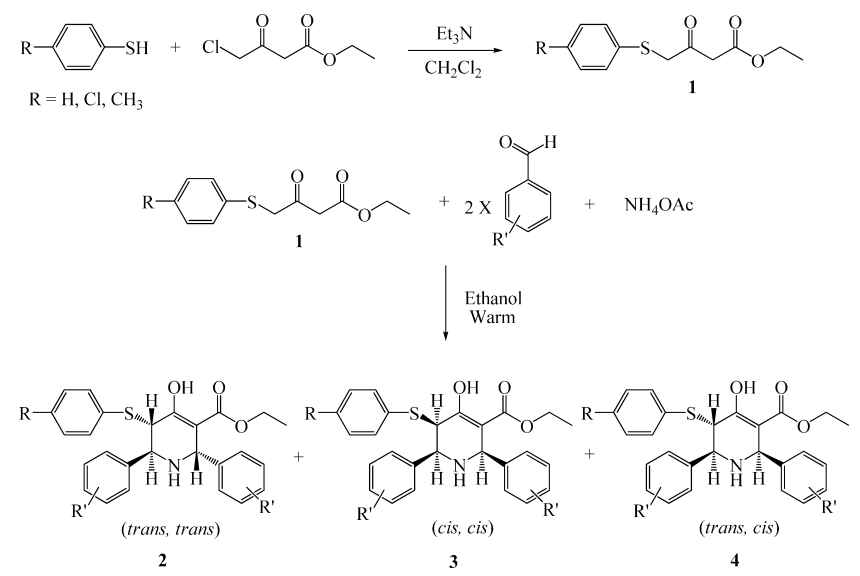

Chart 1. Synthesis of Tetrahydropyridines
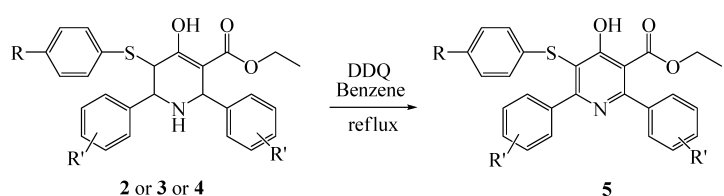

Chart 2. Synthesis of Pentasubstituted Pyridines 
have been screened as a part of our programme to unearth novel heterocyclic leads ${ }^{37-43)}$ for antimycobacterial activities and report the results in this paper.

\section{Results and Discussion}

Chemistry The four-component reaction of ethyl 3-oxo4-(arylsulfanyl)butanoates (1), substituted aromatic aldehydes and ammonium acetate afforded the hitherto unreported ethyl 4-hydroxy-2,6-diaryl-5-(arylsulfanyl)-1,2,5,6-tetrahydro-3-pyridine-carboxylates (2-4) in moderate yields (Chart 1) following the methodology reported by Baliah and Noller for the synthesis of 2,6-diarylpiperidin-4-ones. ${ }^{44)}$ The ethyl 3-oxo-4-(arylsulfanyl)butanoates were prepared by a literature method ${ }^{45)}$ from the reaction of ethyl 4-chloroacetoacetate with substituted thiophenol in the presence of $\mathrm{Et}_{3} \mathrm{~N}$ in $\mathrm{CH}_{2} \mathrm{Cl}_{2}$.

A mixture of ethyl 3-oxo-4-(arylsulfanyl)butanoate, aromatic aldehyde and ammonium acetate in a molar ratio of $1: 2: 1$ in ethanol was gently heated to boiling on a water bath until orange colour appeared and then the reaction mixture was kept aside for $1-2 \mathrm{~d}$ at ambient temperature. After completion of the reaction, the viscous liquid was dissolved in diethyl ether and the compound was isolated as hydrochloride, which was then neutralized with aqueous ammonia. The product, comprising three diastereomeric enols, 2-4 was extracted with ether and the enols separated by column chromatography.

The enols possess three stereocentres and exist in three diastereomeric forms, $\mathbf{2}-\mathbf{4}$ differing in their configurations at 2,5 and 6 positions. In case of entries $1-4,9-12$ and $17-$ 20 where the aryl ring is $p$-substituted, the isomer 2 predominates over 3 and 4. In 2, H-5 and H-6 are trans to each other as evident from their $J$ value of $c a .10 \mathrm{~Hz}$ and $\mathrm{H}-2$ is trans to H-6. In the case of $\mathbf{b}$ and $\mathbf{c}$ (entries 2 and 3), the isomer 4 predominates over $\mathbf{2}$, where the isomer $\mathbf{3}$ is not formed even in traces. In the case of entries $5-7,13-15,21$ and 22 $(\mathbf{3 e}-\mathbf{g}, \mathbf{3} \mathbf{m}-\mathbf{o}, \mathbf{3 u}$ and $\mathbf{3 v})$ where the aryl ring is $o$-substituted, the isomer $\mathbf{3}$ is obtained as the sole product and it is pertinent to note that $\mathrm{H}-5$ and $\mathrm{H}-6$ are in cis relationship. However, in the case of $\mathrm{R}^{\prime}=m-\mathrm{NO}_{2}$ (entries 8, 6 and 23), the isomers $\mathbf{2}$ and $\mathbf{3}$ was obtained as an inseparable mixture, as they differ little in their $R f$ values. Repetition of this reaction also confirmed this observation. In compound 4, H-5 and $\mathrm{H}-$ 2 are respectively trans and cis to H-6. With the range of limited substituents on the aryl rings, it is not possible to discern any firm trend on the influence of the substituents on the product distribution. The structure of the diasteromeric enols was elucidated using NMR spectroscopic data, as illustrated for $\mathbf{2} \mathbf{i}$ and $\mathbf{3 i}$ as representative examples. The relative orientations of the aryl rings at 2 and 6 positions were unambiguously determined by X-ray crystallographic studies (vide infra).

The ${ }^{1} \mathrm{H}-\mathrm{NMR}$ spectrum of $\mathbf{2} \mathbf{i}$ shows a singlet at $4.97 \mathrm{ppm}$ ascribable to H-2 on the basis of its singlet nature and its heteronuclear multiple bond correlations (HMBC) (Fig. 1) with (i) ipso- and ortho-carbons of the phenyl ring at C-2, (ii) C-3 at $96.7 \mathrm{ppm}$, (iii) $\mathrm{C}-4$ at $155.1 \mathrm{ppm}$, (iv) the carbethoxy carbonyl at $169.0 \mathrm{ppm}$, and (v) C-6 at $56.2 \mathrm{ppm}$. The C,H-correlation of $\mathrm{H}-2$ in conjunction with its chemical shift assigns the signal at $56.8 \mathrm{ppm}$ to $\mathrm{C}-2$. From $\mathrm{C}, \mathrm{H}-\mathrm{COSY}$ correlation spectra, the doublet at $3.72 \mathrm{ppm}(J=10.1 \mathrm{~Hz})$ is assigned to $\mathrm{H}-6$. The doublet at $3.82 \mathrm{ppm}(J=10.1 \mathrm{~Hz})$ is due to $\mathrm{H}-5$ as

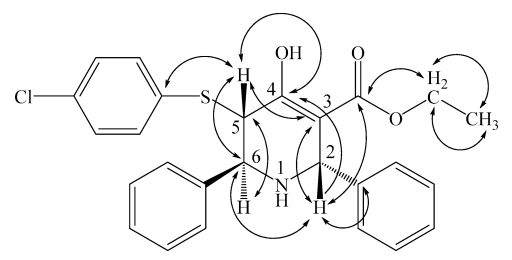

Fig. 1. Selected HMBC Correlations of $\mathbf{2 i}$ (trans, trans)

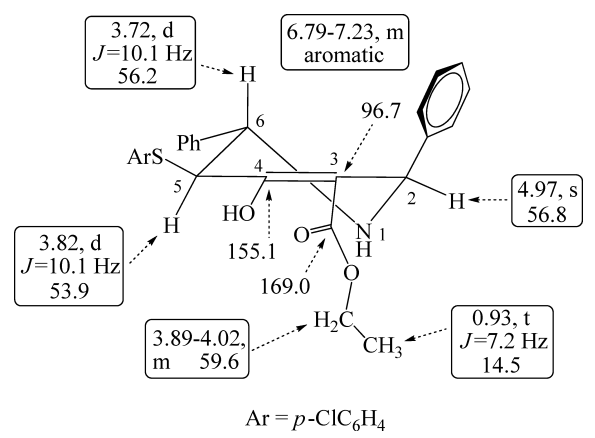

Fig. 2. ${ }^{1} \mathrm{H}$ and ${ }^{13} \mathrm{C}$ Chemical Shifts of $\mathbf{2} \mathbf{i}$ (trans, trans)

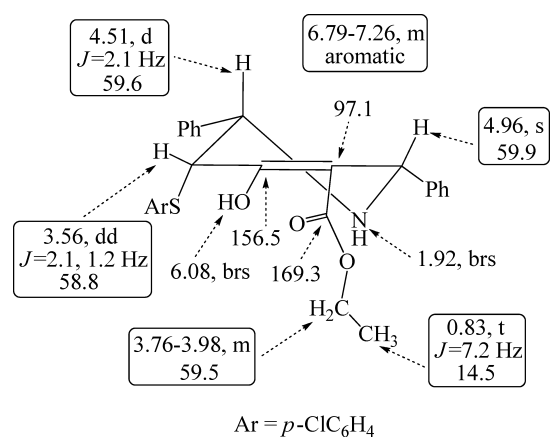

Fig. 3. ${ }^{1} \mathrm{H}$ and ${ }^{13} \mathrm{C}$ Chemical Shifts of $\mathbf{3 i}$ (cis, cis)

evident from its H,H-COSY correlation with H-6 and the signal at $53.9 \mathrm{ppm}$ is assigned to $\mathrm{C}-5$ from $\mathrm{C}, \mathrm{H}-\mathrm{COSY}$ correlation. Further, H-5 shows HMBC correlation with $\mathrm{C}-3$ besides showing correlations with C-4 and C-6. The carbethoxy group affords a triplet at $0.93 \mathrm{ppm}(J=7.2 \mathrm{~Hz})$ and a multiplet at 3.89-4.02 ppm. The NH and aromatic hydrogens appear as a singlet and multiplet at 1.74 and $6.79-7.23 \mathrm{ppm}$ respectively.

The ${ }^{1} \mathrm{H}$ and ${ }^{13} \mathrm{C}$ chemical shifts of $\mathbf{2} \mathbf{i}$ and $\mathbf{3 i}$ differ little. In the case of 3i, H-6 appearing as a doublet at $4.51 \mathrm{ppm}(J=$ $2.1 \mathrm{~Hz}$ ) is deshielded relative to $\mathrm{H}-6$ of $2 \mathbf{i}$. This may probably be taken as an evidence for the stereochemistry of $\mathbf{3 i}$, for which suitable crystals could not be grown and hence X-ray crystallographic studies could not be carried out. Presumably, the two phenyl rings in the case of $2 \mathbf{i}$ shield H-6, while the two phenyl rings in $\mathbf{3 i}$ are far away from H-6 and hence the latter is not shielded. A doublet of doublets at $3.56 \mathrm{ppm}$ $(J=2.1,1.2 \mathrm{~Hz})$ is due to $\mathrm{H}-5$ of $\mathbf{3 i}$, the $J$ values respectively ascribable to vicinal coupling with $\mathrm{H}-6$ and allylic coupling with $\mathrm{OH}$. The $J$ value of $2.1 \mathrm{~Hz}$ discloses their cis relationship as given in Fig. 3. The $\mathrm{NH}$ and $\mathrm{OH}$ hydrogens appear as broad singlets at 1.92 and $6.08 \mathrm{ppm}$ respectively. The ${ }^{1} \mathrm{H}$ and ${ }^{13} \mathrm{C}$ chemical shifts of $\mathbf{2 i}$ and $\mathbf{3 i}$ are depicted in Figs. 2 and 3 respectively. In the case of $\mathbf{4} \mathbf{b}, \mathrm{H}-2$ appears as a singlet at 4.82 ppm and $\mathrm{H}-5, \mathrm{H}-6$ and $\mathrm{CH}_{2}$ of carbethoxy group occur 


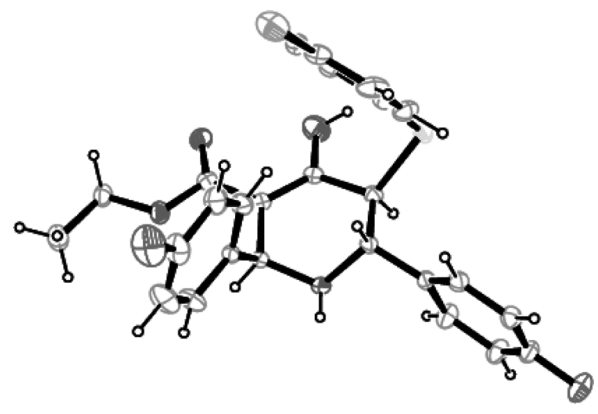

Fig. 4. ORTEP Diagram of $\mathbf{2 k}$

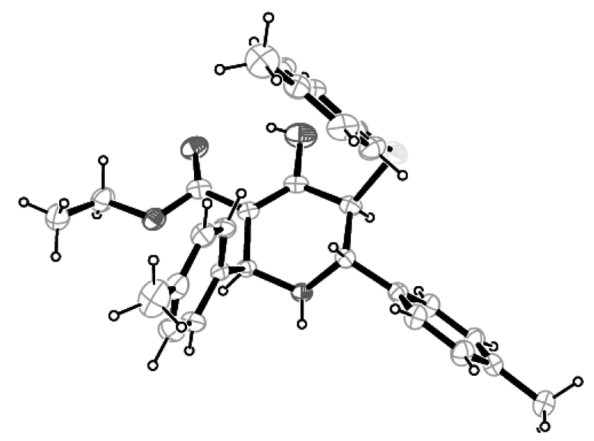

Fig. 5. ORTEP Diagram of $\mathbf{2 t}$

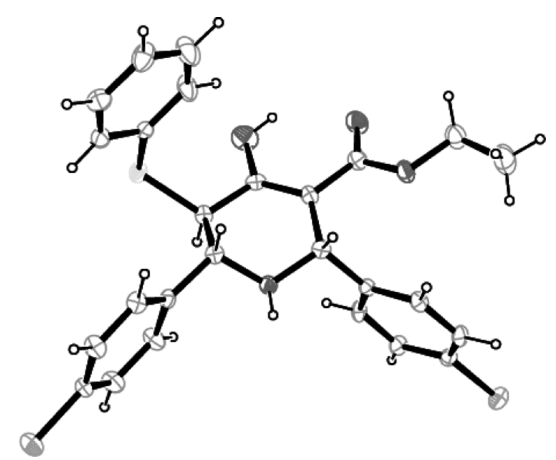

Fig. 6. ORTEP Diagram of $\mathbf{4 b}$

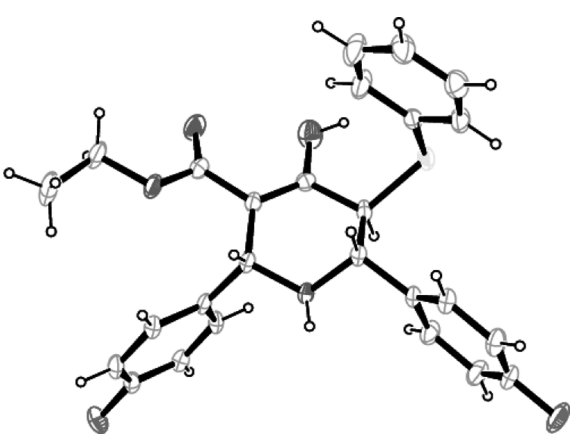

Fig. 7. ORTEP diagram of $\mathbf{4 c}$

as a multiplet at $3.78-3.95 \mathrm{ppm}$. The structures of $\mathbf{2}$ and $\mathbf{4}$ have been fully elucidated from X-ray crystallographic studies $^{46-49)}$ (Figs. 4-7).

Subsequently, the ethyl 4-hydroxy-2,6-diaryl-5-(arylsulfanyl)-1,2,5,6-tetrahydro-3-pyridinecarboxylates (2-4) were dehydrogenated with dichlorodicyanobenzoquinone (DDQ) in refluxing benzene to yield 4-hydroxy-2,6-diaryl-5-(aryl-

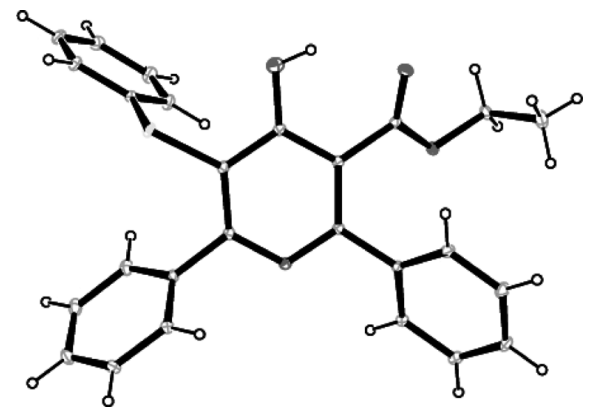

Fig. 8. ORTEP Diagram of $\mathbf{5 a}$

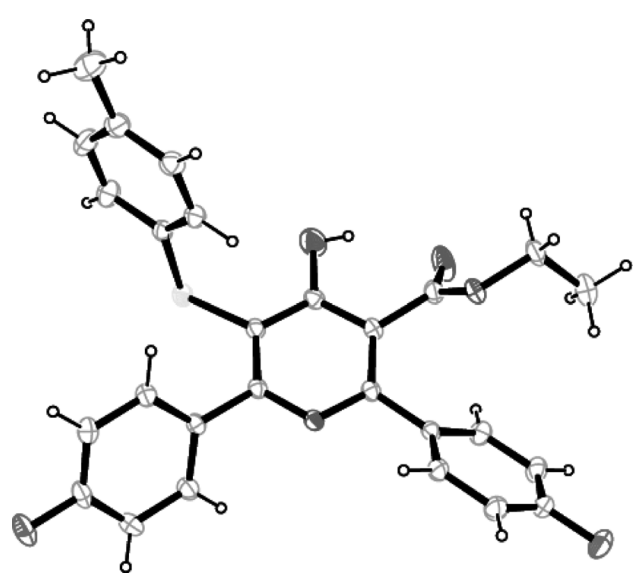

Fig. 9. ORTEP Diagram of $\mathbf{5 r}$

sulfanyl)pyridine-carboxylates (5) in good yield (Chart 2). The major diastereomer in each case was subjected to dehydrogenation with DDQ. After completion of the reaction (TLC), the precipitated DDQ- $\mathrm{H}_{2}$ was filtered off, the solvent removed and the residue crystallized from ethanol to afford the product 5 .

The structure of $\mathbf{5}$ is in accord with its NMR spectroscopic data. For instance, the ${ }^{1} \mathrm{H}-\mathrm{NMR}$ spectrum for 5a has a triplet and a quartet at 0.78 and $3.98 \mathrm{ppm}(J=7.2 \mathrm{~Hz})$ due to the ester function. The aromatic protons appear as a multiplet at $7.06-7.57 \mathrm{ppm}$, while the $\mathrm{OH}$ proton appears as a broad singlet at $6.57 \mathrm{ppm}$. X-ray crystallographic studies ${ }^{50)}$ (Figs. 8 and 9) also confirm the structure deduced from NMR spectroscopic data.

Biology Keto esters (1), tetrahydropyridines (2 -4) and pyridines (5) were screened for their in vitro antimycobacterial activity against Mycobacterium tuberculosis H37Rv (MTB) by agar dilution method for the determination of minimum inhibitory concentration (MIC) in triplicates. The MIC is defined as the minimum concentration of compound required to completely inhibit the bacterial growth. The MIC values of $\mathbf{1}-\mathbf{5}$ along with the standard drugs for comparison are furnished in Table 3.

The keto esters viz. ethyl 3-oxo-4-(arylsulfanyl)butanoates (1) afford MIC $>91.66 \mu \mathrm{M}$ indicating that they are not significantly active against MTB. The tetrahydropyridine esters (2-4) showed good activity with MIC ranging from 6.50$57.94 \mu \mathrm{M}$. All the tetrahydropyridine esters, except 2a, showed good activity with higher activity than the first-line anti-TB drug pyrazinamide (MIC of $50.77 \mu \mathrm{M}$ ). Three compounds, $2 \mathrm{~d}, \mathbf{2 s}$ and $\mathbf{2 t}$, with MIC of 6.81, 6.50 and $6.61 \mu \mathrm{M}$ 
Table 1. Synthesis of Tetrahydropyridines

\begin{tabular}{|c|c|c|c|c|c|c|}
\hline \multirow{2}{*}{ Entry } & \multirow{2}{*}{ Compd. } & \multirow{2}{*}{$\mathrm{R}$} & \multirow{2}{*}{$\mathrm{R}^{\prime}$} & \multicolumn{3}{|c|}{ Yield (\%) } \\
\hline & & & & 2 & 3 & 4 \\
\hline 1 & a & $\mathrm{H}$ & $\mathrm{H}$ & 45 & 7 & - \\
\hline 2 & b & $\mathrm{H}$ & $p-\mathrm{Cl}$ & 6 & - & 54 \\
\hline 3 & c & $\mathrm{H}$ & $p-\mathrm{F}$ & 5 & - & 50 \\
\hline 4 & d & $\mathrm{H}$ & $p-\mathrm{CH}_{3}$ & 42 & 6 & - \\
\hline 5 & e & $\mathrm{H}$ & $o-\mathrm{Cl}$ & - & 52 & - \\
\hline 6 & f & $\mathrm{H}$ & $o-\mathrm{Br}$ & - & 42 & - \\
\hline 7 & g & $\mathrm{H}$ & $o, p-\mathrm{Cl}_{2}$ & - & 55 & - \\
\hline 8 & h & $\mathrm{H}$ & $m-\mathrm{NO}_{2}$ & & $53^{a)}$ & - \\
\hline 9 & $\mathbf{i}$ & $\mathrm{Cl}$ & $\mathrm{H}$ & 46 & 7 & - \\
\hline 10 & $\mathbf{j}$ & $\mathrm{Cl}$ & $p-\mathrm{Cl}$ & 49 & 9 & - \\
\hline 11 & $\mathbf{k}$ & $\mathrm{Cl}$ & $p-\mathrm{F}$ & 28 & 26 & - \\
\hline 12 & 1 & $\mathrm{Cl}$ & $p-\mathrm{CH}_{3}$ & 46 & 6 & - \\
\hline 13 & $\mathbf{m}$ & $\mathrm{Cl}$ & $o-\mathrm{Cl}$ & - & 49 & - \\
\hline 14 & $\mathbf{n}$ & $\mathrm{Cl}$ & $o-\mathrm{Br}$ & - & 40 & - \\
\hline 15 & o & $\mathrm{Cl}$ & $o, p-\mathrm{Cl}_{2}$ & - & 50 & - \\
\hline 16 & p & $\mathrm{Cl}$ & $m-\mathrm{NO}_{2}$ & & $48^{a)}$ & - \\
\hline 17 & $q$ & $\mathrm{CH}_{3}$ & $\mathrm{H}$ & 46 & 8 & - \\
\hline 18 & r & $\mathrm{CH}_{3}$ & $p-\mathrm{Cl}$ & 50 & 7 & - \\
\hline 19 & $\mathbf{s}$ & $\mathrm{CH}_{3}$ & $p-\mathrm{F}$ & 28 & 26 & - \\
\hline 20 & t & $\mathrm{CH}_{3}$ & $p-\mathrm{CH}_{3}$ & 50 & 8 & - \\
\hline 21 & $\mathbf{u}$ & $\mathrm{CH}_{3}$ & $o-\mathrm{Cl}$ & - & 50 & - \\
\hline 22 & $\mathbf{v}$ & $\mathrm{CH}_{3}$ & $o, p-\mathrm{Cl}_{2}$ & - & 45 & - \\
\hline 23 & $\mathbf{w}$ & $\mathrm{CH}_{3}$ & $m-\mathrm{NO}_{2}$ & & $46^{a)}$ & - \\
\hline
\end{tabular}

a) In case of $\mathrm{R}^{\prime}=m-\mathrm{NO}_{2}$ the products were obtained as an inseperable mixture of diastereomers.

Table 2. Synthesis of Pentasubstituted Pyridines

\begin{tabular}{|c|c|c|c|c|}
\hline Entry & Compd. 5 & $\mathrm{R}$ & $\mathrm{R}^{\prime}$ & Yield (\%) \\
\hline 1 & $\mathbf{a}$ & $\mathrm{H}$ & $\mathrm{H}$ & 73 \\
\hline 2 & b & $\mathrm{H}$ & $p-\mathrm{Cl}$ & 84 \\
\hline 3 & c & $\mathrm{H}$ & $p-\mathrm{F}$ & 75 \\
\hline 4 & d & $\mathrm{H}$ & $p-\mathrm{CH}_{3}$ & 72 \\
\hline 5 & e & $\mathrm{H}$ & $o-\mathrm{Cl}$ & 77 \\
\hline 6 & f & $\mathrm{H}$ & $o-\mathrm{Br}$ & 72 \\
\hline 7 & $\mathbf{g}$ & $\mathrm{H}$ & $o, p-\mathrm{Cl}_{2}$ & 82 \\
\hline 8 & h & $\mathrm{H}$ & $m-\mathrm{NO}_{2}$ & 75 \\
\hline 9 & $\mathbf{i}$ & $\mathrm{Cl}$ & $\mathrm{H}^{2}$ & 76 \\
\hline 10 & $\mathbf{j}$ & $\mathrm{Cl}$ & $p-\mathrm{Cl}$ & 87 \\
\hline 11 & $\mathbf{k}$ & $\mathrm{Cl}$ & $p-\mathrm{F}$ & 78 \\
\hline 12 & 1 & $\mathrm{Cl}$ & $p-\mathrm{CH}_{3}$ & 72 \\
\hline 13 & $\mathbf{m}$ & $\mathrm{Cl}$ & $o-\mathrm{Cl}$ & 80 \\
\hline 14 & $\mathbf{n}$ & $\mathrm{Cl}$ & $o-\mathrm{Br}$ & 70 \\
\hline 15 & o & $\mathrm{Cl}$ & $o, p-\mathrm{Cl}_{2}$ & 79 \\
\hline 16 & $\mathbf{p}$ & $\mathrm{CH}_{3}$ & $\mathrm{H}$ & 70 \\
\hline 17 & $q$ & $\mathrm{CH}_{3}$ & $p-\mathrm{Cl}$ & 79 \\
\hline 18 & $\mathbf{r}$ & $\mathrm{CH}_{3}$ & $p$-F & 72 \\
\hline 19 & $\mathbf{s}$ & $\mathrm{CH}_{3}$ & $p-\mathrm{CH}_{3}$ & 70 \\
\hline 20 & $\mathbf{t}$ & $\mathrm{CH}_{3}$ & $o-\mathrm{Cl}$ & 74 \\
\hline 21 & $\mathbf{u}$ & $\mathrm{CH}_{3}$ & $o, p-\mathrm{Cl}_{2}$ & 81 \\
\hline 22 & $\mathbf{v}$ & $\mathrm{CH}_{3}$ & $m-\mathrm{NO}_{2}$ & 78 \\
\hline
\end{tabular}

respectively are more potent than the standard drug ethambutol (MIC of $7.64 \mu \mathrm{M}$ ). The pyridine esters 5 showed good activity with MICs ranging from $1.33-29.24 \mu \mathrm{M}$. All the compounds of series $\mathbf{5}$ are more potent than pyrazinamide (MIC of $50.77 \mu \mathrm{M})$. Ten compounds, 5c- $\mathbf{g}, \mathbf{5 k}, \mathbf{5 p}-\mathbf{r}$ and $\mathbf{5 u}$, with MIC ranging from $1.33-7.09 \mu \mathrm{M}$ are active than the drug ethambutol (MIC of $7.64 \mu \mathrm{M}$ ).

In general, pyridines $\mathbf{5}$ display better activity than the tetrahydropyridine esters (Table 3). Among all the compounds screened, ethyl 2,6-di(2-bromophenyl)-4-hydroxy-5-
Table 3. Anti-mycobacterial Activities of Ketoesters 1, Tetrahydropyridines 2-4 and Pyridines 5

\begin{tabular}{|c|c|c|c|}
\hline Compd. & $(\mathrm{MTB})^{a)}$ & Compd. & $(\mathrm{MTB})^{a)}$ \\
\hline $1 \mathrm{a}$ & $>104.91$ & $4 \mathrm{~b}$ & 24.98 \\
\hline $1 b$ & $>91.66$ & $4 c$ & 13.37 \\
\hline 1c & $>99.08$ & $5 a$ & 29.24 \\
\hline $2 \mathbf{a}$ & 57.94 & $5 b$ & 25.18 \\
\hline 2d & 6.81 & $5 c$ & 6.75 \\
\hline $2 \mathbf{i}$ & 26.82 & $5 d$ & 6.87 \\
\hline $2 j$ & 46.74 & $5 e$ & 6.30 \\
\hline $2 k$ & 12.45 & $5 f$ & 1.33 \\
\hline 21 & 12.65 & $5 \mathrm{~h}$ & 12.08 \\
\hline $2 q$ & 14.03 & $5 \mathbf{i}$ & 27.06 \\
\hline $2 \mathrm{r}$ & 12.15 & $5 j$ & 23.55 \\
\hline $2 s$ & 6.50 & $5 \mathrm{k}$ & 6.29 \\
\hline $2 t$ & 6.61 & 51 & 12.75 \\
\hline $3 e$ & 12.49 & $5 \mathrm{~m}$ & 23.55 \\
\hline $3 f$ & 21.21 & $5 n$ & 10.08 \\
\hline $3 g$ & 21.96 & 50 & 10.42 \\
\hline $3 \mathbf{n}$ & 40.08 & $5 p$ & 7.09 \\
\hline 30 & 41.40 & $5 q$ & 6.13 \\
\hline $3 p$ & $-^{b)}$ & $5 r$ & 3.27 \\
\hline $3 \mathbf{u}$ & 12.15 & $5 s, t$ & $-^{b)}$ \\
\hline $3 \mathbf{v}$ & 42.84 & $5 u$ & 5.40 \\
\hline $3 \mathbf{w}$ & $-^{b)}$ & $5 \mathrm{v}$ & 23.52 \\
\hline \multicolumn{2}{|c|}{ Isoniazid } & \multicolumn{2}{|c|}{0.36} \\
\hline \multicolumn{2}{|c|}{ Rifampicin } & \multicolumn{2}{|c|}{0.12} \\
\hline \multicolumn{2}{|c|}{ Ethambutol } & \multicolumn{2}{|c|}{7.64} \\
\hline \multicolumn{2}{|c|}{ Pyrazinamide } & \multicolumn{2}{|c|}{50.77} \\
\hline
\end{tabular}

a) Minimum inhibitory concentrations $(\mu \mathrm{M})$ against Mycobacterium tuberculosis. b) Not tested.

(phenylsulfanyl)-3-pyridinecarboxylate (5f) showed the maximum activity with MIC of $1.33 \mu \mathrm{M}$. Compared to isoniazid and rifampicin, all the compounds were less active. With regard to structure-activity point of view, most of the pyridines (5) were found to be more potent than the corresponding tetrahydropyridines (2-4). Presumably, the pyridines with enhanced planarity and hence less sterically demanding may have an advantage over tetrahydropyridine enols in penetrating the mycobacterial cell wall. It is found that the presence of (i) methyl group in the aryl ring of 5-arylsulfanyl moiety and (ii) electron-withdrawing groups like halogens in the aryl rings at 2,6-positions enhance the activity.

\section{Conclusion}

The present work describes a rapid access to a series of ethyl 4-hydroxy-2,6-diaryl-5-(arylsulfanyl)-1,2,5,6-tetrahydro-3-pyridinecarboxylates by the four-component reaction of ethyl 3-oxo-4-(arylsulfanyl)butanoate, aromatic aldehyde and ammonium acetate. These tetrahydropyridine esters undergo facile dehydrogenation with DDQ to furnish the highly functionalised pyridines in excellent yields. These novel heterocycles showed good to excellent in vitro activity against Mycobacterium tuberculosis H37Rv. This protocol could be employed for generating a huge library of these biologically important compounds.

\section{Experimental}

General The melting points were taken using open capillary tubes and are uncorrected. ${ }^{1} \mathrm{H},{ }^{13} \mathrm{C}$ and $2 \mathrm{D}$ NMR spectra were recorded on a Bruker (Avance) $300 \mathrm{MHz}$ instrument in $\mathrm{CDCl}_{3}$ using tetramethylsilane (TMS) as internal standard. Chemical shifts are given in parts per million $(\delta$-scale) and the coupling constants are given in Hertz. IR spectra were recorded on a JASCO FT IR instrument $\left(\mathrm{KBr}\right.$ pellet in case of solids and $\mathrm{CHCl}_{3}$ in case of 
viscous liquids). Elemental analyses were performed on a Perkin Elmer 2400 Series II Elemental CHNS analyser. High resolution mass spectra (HRMS) were recorded in Thermo-Scientific mass spectrometer using ESI probe. Column chromatography was performed on silica gel $(230-400$ mesh) using petroleum ether-ethyl acetate $(8: 2 \mathrm{v} / \mathrm{v})$ as eluent. Crystals suitable for X-ray crystallographic studies were obtained by recrystallisation from petroleum ether-ethyl acetate mixture.

Synthesis of Ethyl 4-Hydroxy-2,6-diaryl-5-(arylsulfanyl)-1,2,5,6tetrahydro-3-pyridinecarboxylates (2-4). General Procedure To a solution of ammonium acetate $(0.1 \mathrm{~g}, 1.3 \mathrm{mmol})$ in ethyl alcohol $(30 \mathrm{ml})$, a mixture of ethyl 3-oxo-4-(phenylsulfanyl)butanoate $(0.3 \mathrm{~g}, 1.3 \mathrm{mmol})$ and freshly distilled aromatic aldehyde $(2.6 \mathrm{mmol})$ was added and warmed on a water bath until the solution became orange and the reaction mixture set aside at room temperature for $1-2 \mathrm{~d}$. To this reaction mixture, diethyl ether $(100 \mathrm{ml})$ was added and the solid mass obtained was filtered off. To the clear filtrate, concentrated hydrochloric acid $(2 \mathrm{ml})$ was added with stirring. The solid piperidine hydrochlorides obtained were washed with diethyl ether $(50 \mathrm{ml})$, suspended in acetone, neutralized with ammonia, diluted with icewater, extracted with diethyl ether, and dried over anhydrous sodium sulphate. After removing the solvent, the product mixture was separated in a pure state by column chromatography over silica gel using petroleum ether-ethyl acetate mixture as eluent. The products were further purified by recrystalisation using petroleum ether-ethyl acetate $(8: 2 \mathrm{v} / \mathrm{v})$.

Ethyl 4-Hydroxy-2,6-diphenyl-5-(phenylsulfanyl)-1,2,5,6-tetrahydro-3pyridine-carboxylate (2a): Isolated as white solid; $(0.245 \mathrm{~g}, 45 \%)$; mp 116 $117{ }^{\circ} \mathrm{C} ;{ }^{1} \mathrm{H}-\mathrm{NMR}\left(300 \mathrm{MHz}, \mathrm{CDCl}_{3}\right) \delta_{\mathrm{H}}: 0.92\left(\mathrm{t}, J=7.2 \mathrm{~Hz}, 3 \mathrm{H}, \mathrm{CH}_{3}\right), 3.79$ (d, $J=10.0 \mathrm{~Hz}, 1 \mathrm{H}, \mathrm{H}-6), 3.84$ (d, $J=10.0 \mathrm{~Hz}, 1 \mathrm{H}, \mathrm{H}-5), 3.88-4.12(\mathrm{~m}, 2 \mathrm{H}$, $\left.\mathrm{CH}_{2}\right), 4.97$ (s, 1H, H-2), 6.83-7.28 (m, 15H, aromatic). ${ }^{13} \mathrm{C}-\mathrm{NMR}(75$ $\left.\mathrm{MHz}, \mathrm{CDCl}_{3}\right) \delta_{\mathrm{C}}: 14.5,53.9,56.2,57.0,59.5,96.3,126.7,127.8,128.2$, $128.4,128.5,128.9,129.3,129.6,131.1,135.4,142.1,144.6,155.7,169.0$ IR (KBr) $v_{\max }: 746,1228,1261,1525,1604,1664,2981,3309,3446 \mathrm{~cm}^{-1}$; Anal. Calcd for $\mathrm{C}_{26} \mathrm{H}_{25} \mathrm{NO}_{3} \mathrm{~S}$ : C, 72.36. H, 5.84; N, 3.25; Found: C, 72.30; H, 5.88; N, 3.29.

Ethyl 4-Hydroxy-2,6-diphenyl-5-(phenylsulfanyl)-1,2,5,6-tetrahydro-3pyridine-carboxylate (3a): Isolated as colorless solid; $(0.036 \mathrm{~g}, 7 \%)$; mp $112-114{ }^{\circ} \mathrm{C} ;{ }^{1} \mathrm{H}-\mathrm{NMR}\left(300 \mathrm{MHz}, \mathrm{CDCl}_{3}\right) \delta_{\mathrm{H}}: 0.83\left(\mathrm{t}, J=6.9 \mathrm{~Hz}, 3 \mathrm{H}, \mathrm{CH}_{3}\right)$, $1.72(\mathrm{brs}, 1 \mathrm{H}, \mathrm{NH}), 3.62(\mathrm{~s}, 1 \mathrm{H}, \mathrm{H}-5), 3.85-4.12\left(\mathrm{~m}, 2 \mathrm{H}, \mathrm{CH}_{2}\right), 4.51(\mathrm{~s}$, $1 \mathrm{H}, \mathrm{H}-6), 4.97$ (s, 1H, H-2), 6.07 (br s, $1 \mathrm{H}, \mathrm{OH}), 6.90-7.38(\mathrm{~m}, 15 \mathrm{H}$, aromatic). ${ }^{13} \mathrm{C}-\mathrm{NMR}\left(75 \mathrm{MHz}, \mathrm{CDCl}_{3}\right) \delta_{\mathrm{C}}: 14.2,58.5,59.4,59.6,60.0,97.2$, $127.3,127.8,128.0,128.2,128.4,128.5,129.3,133.2,136.2,140.1,146.3$, 157.3, 169.3. IR (KBr) $v_{\max }: 745,1226,1260,1526,1602,1667,2984$, 3313, $3442 \mathrm{~cm}^{-1}$; Anal. Calcd for $\mathrm{C}_{26} \mathrm{H}_{25} \mathrm{NO}_{3} \mathrm{~S}$ : C, 72.36; H, 5.84; N, 3.25. Found: C, 72.32; H, 5.80; N, 3.31.

Ethyl 2,6-Di(4-chlorophenyl)-4-hydroxy-5-(phenylsulfanyl)-1,2,5,6-tetrahydro-3-pyridinecarboxylate (2b): Isolated as colorless crystals; $(0.038 \mathrm{~g}, 6 \%)$; mp $110-112{ }^{\circ} \mathrm{C} ;{ }^{1} \mathrm{H}-\mathrm{NMR}\left(300 \mathrm{MHz}, \mathrm{CDCl}_{3}\right) \delta_{\mathrm{H}}: 0.94(\mathrm{t}, J=7.2 \mathrm{~Hz}, 3 \mathrm{H}$, $\left.\mathrm{CH}_{3}\right), 3.70(\mathrm{~d}, J=10.0 \mathrm{~Hz}, 1 \mathrm{H}, \mathrm{H}-6), 3.76(\mathrm{~d}, J=10.0 \mathrm{~Hz}, 1 \mathrm{H}, \mathrm{H}-5), 3.89$ $4.02\left(\mathrm{~m}, 2 \mathrm{H}, \mathrm{CH}_{2}\right), 4.91(\mathrm{~s}, 1 \mathrm{H}, \mathrm{H}-2), 6.74-7.45\left(\mathrm{~m}, 13 \mathrm{H}\right.$, aromatic). ${ }^{13} \mathrm{C}-$ NMR $\left.\left(75 \mathrm{MHz}^{\mathrm{CDCl}}\right)_{3}\right) \delta_{\mathrm{C}}: 14.5,53.5,55.6,56.3,59.6,96.0,128.4,129.1$, $129.2,129.6,129.7,129.8,130.6,132.5,134.2,135.6,140.3,143.1,155.5$, 168.8. IR (KBr) $v_{\max }: 738,1230,1261,1529,1604,1664,2987,3313$, $3453 \mathrm{~cm}^{-1}$; Anal. Calcd for $\mathrm{C}_{26} \mathrm{H}_{23} \mathrm{Cl}_{2} \mathrm{NO}_{3} \mathrm{~S}$ : C, 62.40; H, 4.63; N, 2.80 . Found: C, 62.46; H, 4.57; N, 2.84.

Ethyl 2,6-Di(4-chlorophenyl)-4-hydroxy-5-(phenylsulfanyl)-1,2,5,6-tetrahydro-3-pyridinecarboxylate (4b): Isolated as colorless crystals; $(0.340 \mathrm{~g}$, $54 \%)$; mp $158-159{ }^{\circ} \mathrm{C} ;{ }^{1} \mathrm{H}-\mathrm{NMR}\left(300 \mathrm{MHz}, \mathrm{CDCl}_{3}\right) \delta_{\mathrm{H}}: 0.82(\mathrm{t}, J=7.2 \mathrm{~Hz}$, $\left.3 \mathrm{H}, \mathrm{CH}_{3}\right), 3.78-3.95\left(\mathrm{~m}, 4 \mathrm{H}, \mathrm{H}-5, \mathrm{H}-6, \mathrm{CH}_{2}\right), 4.82$ (s, 1H, H-2), 7.11$7.23\left(\mathrm{~m}, 13 \mathrm{H}\right.$, aromatic). ${ }^{13} \mathrm{C}-\mathrm{NMR}\left(75 \mathrm{MHz} \mathrm{CDCl}_{3}\right) \delta_{\mathrm{C}}: 14.2,55.8,59.4$, $59.5,62.1,98.3,128.3,128.4,128.9,129.5,129.6,129.8,132.7,132.8$, $132.9,134.2,140.1,145.3,155.5,168.9$. IR (KBr) $v_{\max }: 740,1232,1260$, 1527, 1601, 1665, 2985, 3310, 3451 cm ${ }^{-1}$; Anal. Calcd for $\mathrm{C}_{26} \mathrm{H}_{23} \mathrm{Cl}_{2} \mathrm{NO}_{3} \mathrm{~S}$ : C, 62.40; H, 4.63; N, 2.80. Found: C, 62.36; H, 4.69; N, 2.86.

Ethyl 2,6-Di(4-fluorophenyl)-4-hydroxy-5-(phenylsulfanyl)-1,2,5,6-tetrahydro-3-pyridinecarboxylate (2c): Isolated as colorless crystals; $(0.030 \mathrm{~g}, 5 \%)$; mp $109-110{ }^{\circ} \mathrm{C} ;{ }^{1} \mathrm{H}-\mathrm{NMR}\left(300 \mathrm{MHz}, \mathrm{CDCl}_{3}\right) \delta_{\mathrm{H}}: 0.91(\mathrm{t}, J=7.2 \mathrm{~Hz}, 3 \mathrm{H}$, $\left.\mathrm{CH}_{3}\right), 3.73$ (d, $\left.J=9.5 \mathrm{~Hz}, 1 \mathrm{H}, \mathrm{H}-6\right), 3.82$ (d, $\left.J=9.5 \mathrm{~Hz}, 1 \mathrm{H}, \mathrm{H}-5\right), 3.87-4.09$ $\left(\mathrm{m}, 2 \mathrm{H}, \mathrm{CH}_{2}\right), 4.95(\mathrm{~s}, 1 \mathrm{H}, \mathrm{H}-2), 6.77-7.56\left(\mathrm{~m}, 13 \mathrm{H}\right.$, aromatic). ${ }^{13} \mathrm{C}-\mathrm{NMR}$ $\left(75 \mathrm{MHz} \mathrm{CDCl}_{3}\right) \delta_{\mathrm{C}}: 14.6,53.6,55.4,56.7,59.7,96.0,115.0,115.2,115.5$, $115.8,128.3,129.5,129.7,129.9,130.2,132.1,133.1,136.1,143.7,160.3$, $161.2,163.5,165.2,155.5,168.3$. IR (KBr) $v_{\max }: 737,1231,1263,1530$, 1604, 1665, 2989, 3314, 3451 cm $\mathrm{cm}^{-1}$ Anal. Calcd for $\mathrm{C}_{26} \mathrm{H}_{23} \mathrm{~F}_{2} \mathrm{NO}_{3} \mathrm{~S}$ : C, 66.79; H, 4.96; N, 3.00. Found: C, 66.85; H, 4.91; N, 3.07.

Ethyl 2,6-Di(4-fluorophenyl)-4-hydroxy-5-(phenylsulfanyl)-1,2,5,6-tetrahydro-3-pyridinecarboxylate (4c): Isolated as colorless crystals; $(0.295 \mathrm{~g}$,

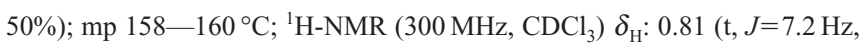
$\left.3 \mathrm{H}, \mathrm{CH}_{3}\right), 3.77-3.95\left(\mathrm{~m}, 3 \mathrm{H}, \mathrm{H}-5, \mathrm{H}-6, \mathrm{CH}_{2}\right), 4.83$ (s, 1H, H-2), 6.89$7.22\left(\mathrm{~m}, 13 \mathrm{H}\right.$, aromatic). ${ }^{13} \mathrm{C}-\mathrm{NMR}\left(75 \mathrm{MHz}, \mathrm{CDCl}_{3}\right) \delta_{\mathrm{C}}: 14.5,56.0,59.3$, $59.4,62.0,98.7,114.9,115.1,115.4,115.7,128.2,129.4,129.6,129.7$, $130.0,132.9,133.0,137.5,142.5,160.6,161.2,163.8,164.5,155.5,169.0$. IR $(\mathrm{KBr}) v_{\max }: 749,1232,1262,1528,1602,1668,2987,3309,3455 \mathrm{~cm}^{-1}$; Anal. Calcd for $\mathrm{C}_{26} \mathrm{H}_{23} \mathrm{~F}_{2} \mathrm{NO}_{3} \mathrm{~S}$ : C, 66.79; H, 4.96; N, 3.00. Found: C, 66.75; $\mathrm{H}, 4.92 ; \mathrm{N}, 3.05$.

Ethyl 4-Hydroxy-2,6-di(4-methylphenyl)-5-(phenylsulfanyl)-1,2,5,6-tetrahydro-3-pyridinecarboxylate (2d): Isolated as colorless crystals; $(0.242 \mathrm{~g}$, $42 \%)$; mp $128-129^{\circ} \mathrm{C} ;{ }^{1} \mathrm{H}-\mathrm{NMR}\left(300 \mathrm{MHz} \mathrm{CDCl}_{3}\right) \delta_{\mathrm{H}}: 0.94(\mathrm{t}, J=7.2 \mathrm{~Hz}$, $\left.3 \mathrm{H}, \mathrm{CH}_{3}\right), 2.27\left(\mathrm{~s}, 1 \mathrm{H}, \mathrm{CH}_{3}\right), 2.32\left(\mathrm{~s}, 1 \mathrm{H}, \mathrm{CH}_{3}\right), 3.76(\mathrm{~d}, J=10.2 \mathrm{~Hz}, 1 \mathrm{H}, \mathrm{H}-$ 6), $3.82(\mathrm{~d}, J=10.2 \mathrm{~Hz}, 1 \mathrm{H}, \mathrm{H}-5), 3.87-4.03\left(\mathrm{~m}, 2 \mathrm{H}, \mathrm{CH}_{2}\right), 4.93(\mathrm{~s}, 1 \mathrm{H}, \mathrm{H}-$ 2), $6.70-7.43\left(\mathrm{~m}, 13 \mathrm{H}\right.$, aromatic). ${ }^{13} \mathrm{C}-\mathrm{NMR}\left(75 \mathrm{MHz}, \mathrm{CDCl}_{3}\right) \delta_{\mathrm{C}}: 14.6$, $54.0,55.8,56.7,59.5,96.6,127.7,128.4,128.9,129.2,129.5,129.6,131.2$, $135.4,136.1,138.1,139.3,141.7,155.7,169.1$. IR (KBr) $v_{\max }: 738,1229$, 1260, 1520, 1601, 1667, 2988, 3306, 3449 $\mathrm{cm}^{-1}$; Anal. Calcd for $\mathrm{C}_{28} \mathrm{H}_{29}$ $\mathrm{NO}_{3}$ S: C, 73.17; H, 6.36; N, 3.05. Found: C, 73.12; H, 6.30; N, 3.00.

Ethyl 4-Hydroxy-2,6-di(4-methylphenyl)-5-(phenylsulfanyl)-1,2,5,6-tetrahydro-3-pyridinecarboxylate (3d): Isolated as colorless crystals; $(0.035 \mathrm{~g}, 6 \%)$; mp $111-112{ }^{\circ} \mathrm{C} ;{ }^{1} \mathrm{H}-\mathrm{NMR}\left(300 \mathrm{MHz}, \mathrm{CDCl}_{3}\right) \delta_{\mathrm{H}}: 0.85(\mathrm{t}, J=6.9 \mathrm{~Hz}, 3 \mathrm{H}$, $\left.\mathrm{CH}_{3}\right), 1.89($ br s, $1 \mathrm{H}, \mathrm{NH}), 2.29\left(\mathrm{~s}, 1 \mathrm{H}, \mathrm{CH}_{3}\right), 2.34\left(\mathrm{~s}, 1 \mathrm{H}, \mathrm{CH}_{3}\right), 3.60(\mathrm{~d}, J=$ $1.8 \mathrm{~Hz}, 1 \mathrm{H}, \mathrm{H}-5), 3.84-4.10\left(\mathrm{~m}, 2 \mathrm{H}, \mathrm{CH}_{2}\right), 4.44(\mathrm{~d}, J=1.8 \mathrm{~Hz}, 1 \mathrm{H}, \mathrm{H}-6)$, 4.93 (s, $1 \mathrm{H}, \mathrm{H}-2), 6.00$ (br s, $1 \mathrm{H}, \mathrm{OH}), 6.89-7.47$ (m, $13 \mathrm{H}$, aromatic). ${ }^{13} \mathrm{C}-$ NMR $\left(75 \mathrm{MHz}, \mathrm{CDCl}_{3}\right) \delta_{\mathrm{C}}: 13.8,21.1,21.2,58.2,58.9,58.9,59.1,96.4$, $127.0,127.2,127.4,127.9,128.5,128.7,128.8,132.8,136.3,136.7,136.9$, 143.0, 156.7, 169.0. IR (KBr) $v_{\max }: 740,1225,1262,1526,1607,1662$ 2984, 3312, $3445 \mathrm{~cm}^{-1}$; Anal. Calcd for $\mathrm{C}_{28} \mathrm{H}_{29} \mathrm{NO}_{3} \mathrm{~S}: \mathrm{C}, 73.17 ; \mathrm{H}, 6.36$; N, 3.05. Found: C, 73.24; H, 6.31; N, 3.11.

Ethyl 2,6-Di(2-chlorophenyl)-4-hydroxy-5-(phenylsulfanyl)-1,2,5,6-tetrahydro-3-pyridinecarboxylate (3e): Isolated as colorless crystals; $(0.325 \mathrm{~g}$, $52 \%)$; mp 141- $142{ }^{\circ} \mathrm{C}$; ${ }^{1} \mathrm{H}-\mathrm{NMR}\left(300 \mathrm{MHz}, \mathrm{CDCl}_{3}\right) \delta_{\mathrm{H}}: 0.84(\mathrm{t}, J=7.2 \mathrm{~Hz}$, $\left.3 \mathrm{H}, \mathrm{CH}_{3}\right), 1.94$ (brs, $\left.1 \mathrm{H}, \mathrm{NH}\right), 3.77-3.97\left(\mathrm{~m}, 2 \mathrm{H}, \mathrm{CH}_{2}\right), 3.98-4.05(\mathrm{~m}$, 1H, H-5), 4.81 (s, 1H, H-6), 5.55 (s, 1H, H-2), 6.18 (br s, 1H, OH), 6.93$7.55\left(\mathrm{~m}, 13 \mathrm{H}\right.$, aromatic). ${ }^{13} \mathrm{C}-\mathrm{NMR}\left(75 \mathrm{MHz}, \mathrm{CDCl}_{3}\right) \delta_{\mathrm{C}}: 13.6,54.1,55.0$, $56.3,59.0,95.3,126.7,127.1,127.3,127.9,128.6,128.7,128.8,128.9$, $129.9,132.0,132.4,133.7,135.6,136.9,143.4,157.1,168.5 . \mathrm{IR}(\mathrm{KBr}) v_{\max }$ : 740, 1221, 1265, 1523, 1612, 1669, 2989, 3319, $3447 \mathrm{~cm}^{-1}$; Anal. Calcd for $\mathrm{C}_{26} \mathrm{H}_{23} \mathrm{Cl}_{2} \mathrm{NO}_{3} \mathrm{~S}$ : C, 62.40; H, 4.63; N, 2.80. Found: C, 62.47; H, 4.58; N, 2.86 .

Ethyl 2,6-Di(2-bromophenyl)-4-hydroxy-5-(phenylsulfanyl)-1,2,5,6-tetrahydro-3-pyridinecarboxylate (3f): Isolated as colorless crystals; $(0.310 \mathrm{~g}$, $42 \%)$; mp 135-136 ${ }^{\circ} \mathrm{C} ;{ }^{1} \mathrm{H}-\mathrm{NMR}\left(300 \mathrm{MHz}, \mathrm{CDCl}_{3}\right) \delta_{\mathrm{H}}: 0.90(\mathrm{t}, J=7.2 \mathrm{~Hz}$, $\left.3 \mathrm{H}, \mathrm{CH}_{3}\right), 1.97$ (br s, 1H, NH), 3.88-4.07 (m, 2H, $\left.\mathrm{CH}_{2}\right), 4.20(\mathrm{~s}, 1 \mathrm{H}, \mathrm{H}-5)$, $4.77(\mathrm{~s}, 1 \mathrm{H}, \mathrm{H}-6), 5.48(\mathrm{~s}, 1 \mathrm{H}, \mathrm{H}-2), 6.98-7.58(\mathrm{~m}, 13 \mathrm{H}$, aromatic), $12.3(\mathrm{~s}$, $1 \mathrm{H}, \mathrm{OH}) .{ }^{13} \mathrm{C}-\mathrm{NMR}\left(75 \mathrm{MHz}, \mathrm{CDCl}_{3}\right) \delta_{\mathrm{C}}: 13.2,52.8,56.4,58.6,60.2$, $100.6,109.0,122.7,126.8,126.9,127.7,128.3,128.8,130.1,131.8,133.3$, $133.9,134.3,134.8,134.9,135.2,142.3,157.1,170.5$. IR (KBr) $v_{\max }: 741$, 1224, 1262, 1520, 1614, 1667, 2990, 3317, $3445 \mathrm{~cm}^{-1}$; Anal. Calcd for $\mathrm{C}_{26} \mathrm{H}_{23} \mathrm{Br}_{2} \mathrm{NO}_{3} \mathrm{~S}$ : C, 52.99; H, 3.93; N, 2.38. Found C, 53.05; H, 4.01; N, 2.30 .

Ethyl 2,6-Di(2,4-dichlorophenyl)-4-hydroxy-5-(phenylsulfanyl)-1,2,5,6tetrahydro-3-pyridinecarboxylate $\mathbf{3 g})$ : Isolated as pale yellow solid; $(0.395$ $\mathrm{g}, 55 \%)$; mp $150-151{ }^{\circ} \mathrm{C} ;{ }^{1} \mathrm{H}-\mathrm{NMR}\left(300 \mathrm{MHz}, \mathrm{CDCl}_{3}\right) \delta_{\mathrm{H}}: 0.96(\mathrm{t}, J=$ $\left.7.2 \mathrm{~Hz}, 3 \mathrm{H}, \mathrm{CH}_{3}\right), 1.88($ br s, $1 \mathrm{H}, \mathrm{NH}), 3.92-4.09\left(\mathrm{~m}, 2 \mathrm{H}, \mathrm{CH}_{2}\right), 4.15(\mathrm{~s}$, $1 \mathrm{H}, \mathrm{H}-5), 4.74$ (s, 1H, H-6), 5.44 (s, 1H, H-2), $7.02-7.31$ (m, 9H, aromatic), $7.38(\mathrm{~d}, J=1.8 \mathrm{~Hz}, 1 \mathrm{H}$, aromatic), $7.47(\mathrm{~d}, J=8.4 \mathrm{~Hz}, 1 \mathrm{H}$, aromatic), $12.3(\mathrm{~s}, 1 \mathrm{H}, \mathrm{OH}) .{ }^{13} \mathrm{C}-\mathrm{NMR}\left(75 \mathrm{MHz}, \mathrm{CDCl}_{3}\right) \delta_{\mathrm{C}}: 13.5,52.8,52.9,56.1$, $60.7,100.6,126.7,127.3,127.6,128.4,128.6,129.9,130.6,132.1,132.9$, $133.3,133.9,134.3,134.8,134.9,140.3,170.4,170.6$. IR (KBr) $v_{\max }: 739$, $1225,1266,1522,1618,1668,2988,3317,3441 \mathrm{~cm}^{-1}$; Anal. Calcd for $\mathrm{C}_{26} \mathrm{H}_{21} \mathrm{Cl}_{4} \mathrm{NO}_{3} \mathrm{~S}: \mathrm{C}, 54.85 ; \mathrm{H}, 3.72 ; \mathrm{N}, 2.46$. Found: C, 54.78; H, 3.80; N, 2.39 .

Ethyl 4-Hydroxy-2,6-di(3-nitrophenyl)-5-(phenylsulfanyl)-1,2,5,6-tetrahydro-3-pyridinecarboxylate (2h and $\mathbf{3 h}$ ) (Appears as Mixture in a Ratio 1 : $0.62)$ : Isolated as viscous liquid; $(0.350 \mathrm{~g}, 53 \%)$; ${ }^{1} \mathrm{H}-\mathrm{NMR}(300 \mathrm{MHz}$, $\left.\mathrm{CDCl}_{3}\right) \delta_{\mathrm{H}}: 0.95-1.05\left(\mathrm{~m}, \mathrm{CH}_{3}\right), 3.94-4.16\left(\mathrm{~m}, \mathrm{CH}_{2}\right), 4.26-4.37(\mathrm{~m}, \mathrm{H}-$ 5), $4.52-5.13$ (m, H-6, H-2), 7.06-8.20 (m, aromatic), $12.6(\mathrm{~s}, \mathrm{OH}), 12.9$ (s, OH). HR-MS (ESI) $\mathrm{m} / z$ Calcd for $\mathrm{C}_{26} \mathrm{H}_{23} \mathrm{~N}_{3} \mathrm{O}_{7} \mathrm{~S}: 521.1257$; Found, $520.2402(\mathrm{M}-1)$.

Ethyl 5-[(4-Chlorophenyl)sulfanyl]-4-hydroxy-2,6-diphenyl-1,2,5,6-tetrahydro-3-pyridinecarboxylate (2i): Isolated as colorless crystals; $(0.236 \mathrm{~g}, 46 \%)$; mp $121-122{ }^{\circ} \mathrm{C} ;{ }^{1} \mathrm{H}-\mathrm{NMR}\left(300 \mathrm{MHz}, \mathrm{CDCl}_{3}\right) \delta_{\mathrm{H}}: 0.93(\mathrm{t}, J=7.2 \mathrm{~Hz}, 3 \mathrm{H}$, $\left.\mathrm{CH}_{3}\right), 1.74(\mathrm{~s}, 1 \mathrm{H}, \mathrm{NH}), 3.72(\mathrm{~d}, J=10.1 \mathrm{~Hz}, 1 \mathrm{H}, \mathrm{H}-6), 3.82$ (d, $J=10.1 \mathrm{~Hz}$, 
1H, H-5), 3.89-4.02 (m, 2H, $\left.\mathrm{CH}_{2}\right), 4.97(\mathrm{~s}, 1 \mathrm{H}, \mathrm{H}-2), 6.79-7.23(\mathrm{~m}, 14 \mathrm{H}$ aromatic). ${ }^{13} \mathrm{C}-\mathrm{NMR}\left(75 \mathrm{MHz}, \mathrm{CDCl}_{3}\right) \delta_{\mathrm{C}}: 14.5,53.9,56.2,56.8,59.6,96.7$, $127.0,127.7,128.2,128.4,128.6,129.0,129.2,129.6,135.8,137.1,141.8$ 144.4, 155.1, 169.0. IR (KBr) $v_{\max }: 740,1230,1261,1529,1604,1664$, 2987, 3313, $3453 \mathrm{~cm}^{-1}$; Anal. Calcd for $\mathrm{C}_{26} \mathrm{H}_{24} \mathrm{ClNO}_{3} \mathrm{~S}: \mathrm{C}, 67.01 ; \mathrm{H}, 5.19$; N, 3.01. Found: C, 67.08; H, 5.25; N, 3.06.

Ethyl 5-[(4-Chlorophenyl)sulfanyl]-4-hydroxy-2,6-diphenyl-1,2,5,6-tetrahydro-3-pyridinecarboxylate (3i): Isolated as colorless crystals; $(0.036 \mathrm{~g}, 7 \%)$; mp $115-117^{\circ} \mathrm{C} ;{ }^{1} \mathrm{H}-\mathrm{NMR}\left(300 \mathrm{MHz}, \mathrm{CDCl}_{3}\right) \delta_{\mathrm{H}}: 0.83(\mathrm{t}, J=7.2 \mathrm{~Hz}, 3 \mathrm{H}$, $\mathrm{CH}_{3}$ ), 1.92 (br s, $\left.1 \mathrm{H}, \mathrm{NH}\right), 3.56$ (dd, $\left.J=2.1,1.2 \mathrm{~Hz}, 1 \mathrm{H}, \mathrm{H}-5\right), 3.76-3.98$ $\left(\mathrm{m}, 2 \mathrm{H}, \mathrm{CH}_{2}\right), 4.51$ (d, $\left.J=2.1 \mathrm{~Hz}, 1 \mathrm{H}, \mathrm{H}-6\right), 4.96$ (s, 1H, H-2), 6.08 (br s, 1H, $\mathrm{OH}), 6.79-7.26\left(\mathrm{~m}, 14 \mathrm{H}\right.$, aromatic). ${ }^{13} \mathrm{C}-\mathrm{NMR}\left(75 \mathrm{MHz}^{\mathrm{CDCl}} \mathrm{CDC}_{3}\right) \delta_{\mathrm{C}}: 14.5$, $58.8,59.5,59.6,59.9,97.1,127.3,127.8,128.0,128.4,128.5,128.6,129.4$, $134.2,134.6,140.0,146.2,156.5,169.3$. IR (KBr) $v_{\max }: 743,1235,1262$, 1526, 1601, 1668, 2992, 3318, $3446 \mathrm{~cm}^{-1}$; Anal. Calcd for $\mathrm{C}_{26} \mathrm{H}_{24} \mathrm{ClNO}_{3} \mathrm{~S}$ : C, 67.01; H, 5.19; N, 3.01. Found: C, 66.97; H, 5.13; N, 3.08

Ethyl 2,6-Di(4-chlorophenyl)-5-[(4-chlorophenyl)sulfanyl]-4-hydroxy1,2,5,6-tetrahydro-3-pyridinecarboxylate $(\mathbf{2} \mathbf{j})$ : Isolated as colorless crystals; $(0.290 \mathrm{~g}, 49 \%) ; \mathrm{mp} 119-120{ }^{\circ} \mathrm{C} ;{ }^{1} \mathrm{H}-\mathrm{NMR}\left(300 \mathrm{MHz}, \mathrm{CDCl}_{3}\right) \delta_{\mathrm{H}}: 0.96(\mathrm{t}$, $\left.J=7.2 \mathrm{~Hz}, 3 \mathrm{H}, \mathrm{CH}_{3}\right), 3.63(\mathrm{~d}, J=9.9 \mathrm{~Hz}, 1 \mathrm{H}, \mathrm{H}-6), 3.74(\mathrm{~d}, J=9.9 \mathrm{~Hz}, 1 \mathrm{H}$, $\mathrm{H}-5), 3.91-4.03\left(\mathrm{~m}, 2 \mathrm{H}, \mathrm{CH}_{2}\right), 4.91(\mathrm{~s}, 1 \mathrm{H}, \mathrm{H}-2), 6.73-7.37(\mathrm{~m}, 12 \mathrm{H}$, aromatic). ${ }^{13} \mathrm{C}-\mathrm{NMR}\left(75 \mathrm{MHz}, \mathrm{CDCl}_{3}\right) \delta_{\mathrm{C}}: 14.2,53.5,55.7,56.1,59.6,96.5$, $128.4,128.9,129.0,129.2,129.7,129.9,132.7,134.4,136.1,137.0,140.1$, 143.0, 155.0, 168.7. IR (KBr) $v_{\max }: 736,1236,1260,1525,1602,1665$, 2984, 3310, $3450 \mathrm{~cm}^{-1}$; Anal. Calcd for $\mathrm{C}_{26} \mathrm{H}_{22} \mathrm{Cl}_{3} \mathrm{NO}_{3} \mathrm{~S}$ : C, 58.38; H, 4.15; N, 2.62. Found: C, 58.31; H, 4.20; N, 2.56.

Ethyl 2,6-Di(4-chlorophenyl)-5-[(4-chlorophenyl)sulfanyl]-4-hydroxy1,2,5,6-tetrahydro-3-pyridinecarboxylate $(\mathbf{3 j})$ : Isolated as colorless crystals; $(0.053 \mathrm{~g}, 9 \%) ; \mathrm{mp} 109-110{ }^{\circ} \mathrm{C} ;{ }^{1} \mathrm{H}-\mathrm{NMR}\left(300 \mathrm{MHz}, \mathrm{CDCl}_{3}\right) \delta_{\mathrm{H}}: 0.89(\mathrm{t}$ $\left.J=7.2 \mathrm{~Hz}, 3 \mathrm{H}, \mathrm{CH}_{3}\right), 1.92$ (br s, $\left.1 \mathrm{H}, \mathrm{NH}\right), 3.54$ (s, $\left.1 \mathrm{H}, \mathrm{H}-5\right), 3.80-3.99$ (m, $2 \mathrm{H}, \mathrm{CH}_{2}$ ), 4.46 (d, J=1.8 Hz, 1H, H-6), 4.93 (s, 1H, H-2), 6.11 (br s, $1 \mathrm{H}$, $\mathrm{OH}), 6.87-7.28\left(\mathrm{~m}, 12 \mathrm{H}\right.$, aromatic). ${ }^{13} \mathrm{C}-\mathrm{NMR}\left(75 \mathrm{MHz}, \mathrm{CDCl}_{3}\right) \delta_{\mathrm{C}}: 14.3$, 58.4, 59.1, 59.2, 59.6, 96.6, 128.6, 128.7, 129.1, 129.5, 129.8, 133.0, 133.9, $134.1,134.6,138.4,144.7,156.5,169.0$. IR (KBr) $v_{\max }: 739,1231,1262$, 1527, 1602, 1660, 2989, 3315, $3451 \mathrm{~cm}^{-1}$; Anal. Calcd for $\mathrm{C}_{26} \mathrm{H}_{22} \mathrm{Cl}_{3} \mathrm{NO}_{3} \mathrm{~S}$ : C, 58.38; H, 4.15; N, 2.62. Found: C, 58.45; H, 4.21; N, 2.66.

Ethyl 5-[(4-Chlorophenyl)sulfanyl]-2,6-di(4-fluorophenyl)-4-hydroxy1,2,5,6-tetrahydro-3-pyridinecarboxylate $(\mathbf{2 k})$ : Isolated as colorless crystals $(0.155 \mathrm{~g}, 28 \%) ; \mathrm{mp} 129-130{ }^{\circ} \mathrm{C} ;{ }^{1} \mathrm{H}-\mathrm{NMR}\left(300 \mathrm{MHz}, \mathrm{CDCl}_{3}\right) \delta_{\mathrm{H}}: 0.95(\mathrm{t}$, $\left.J=7.2 \mathrm{~Hz}, 3 \mathrm{H}, \mathrm{CH}_{3}\right), 3.66$ (d, $\left.J=10.1 \mathrm{~Hz}, 1 \mathrm{H}, \mathrm{H}-6\right), 3.74$ (d, $J=10.1 \mathrm{~Hz}, 1 \mathrm{H}$, $\mathrm{H}-5), 3.91-4.02\left(\mathrm{~m}, 2 \mathrm{H}, \mathrm{CH}_{2}\right), 4.93(\mathrm{~s}, 1 \mathrm{H}, \mathrm{H}-2), 6.73-7.30(\mathrm{~m}, 12 \mathrm{H}$, aromatic). ${ }^{13} \mathrm{C}-\mathrm{NMR}\left(75 \mathrm{MHz}, \mathrm{CDCl}_{3}\right) \delta_{\mathrm{C}}: 14.5,53.9,55.5,56.1,59.6,96.8$, $114.9,115.1,115.8,116.0,129.0,129.1,129.5,129.8,129.9,130.0,136.0$ $137.1,137.5,137.6,140.3,140.4,160.3,161.2,163.5,164.5,155.0,168.8$ IR (KBr) $v_{\max }: 735,1230,1262,1521,1606,1664,2988,3314,3445 \mathrm{~cm}^{-1}$; Anal. Calcd for $\mathrm{C}_{26} \mathrm{H}_{22} \mathrm{ClF}_{2} \mathrm{NO}_{3} \mathrm{~S}$ : C, 62.21; H, 4.42; N, 2.79. Found: C, 62.17; H, 4.37; N, 2.85 .

Ethyl 5-[(4-Chlorophenyl)sulfanyl]-2,6-di(4-fluorophenyl)-4-hydroxy1,2,5,6-tetrahydro-3-pyridinecarboxylate (3k): Isolated as colorless crystals; $(0.144 \mathrm{~g}, 26 \%) ; \mathrm{mp} 112-113{ }^{\circ} \mathrm{C} ;{ }^{1} \mathrm{H}-\mathrm{NMR}\left(300 \mathrm{MHz}, \mathrm{CDCl}_{3}\right) \delta_{\mathrm{H}}: 0.88(\mathrm{t}$, $J=7.2 \mathrm{~Hz}, 3 \mathrm{H}, \mathrm{CH}_{3}$ ), 1.79 (br s, $\left.1 \mathrm{H}, \mathrm{NH}\right), 3.52$ (d, $1 \mathrm{H}, J=1.8 \mathrm{~Hz}, \mathrm{H}-5$ ), $3.81-4.00\left(\mathrm{~m}, 1 \mathrm{H}, \mathrm{CH}_{2}\right), 4.47(\mathrm{~d}, 1 \mathrm{H}, J=1.8 \mathrm{~Hz}, \mathrm{H}-6), 4.94(\mathrm{~s}, 1 \mathrm{H}, \mathrm{H}-2)$, 6.85-7.30 (m, 12H, aromatic). ${ }^{13} \mathrm{C}-\mathrm{NMR}\left(75 \mathrm{MHz}, \mathrm{CDCl}_{3}\right) \delta_{\mathrm{C}}: 14.3,58.7$, $59.0,59.2,59.5,96.6,115.1,115.3,115.4,115.6,129.3,129.4,129.5$, $129.8,130.0,134.3,134.4,134.5,135.7,135.8,141.9,142.0,160.7,161.0$, $163.9,164.2,156.5,169.1$. IR (KBr) $v_{\max }: 738,1236,1260,1524,1601$, 1662, 2985, 3310, $3447 \mathrm{~cm}^{-1}$; Anal. Calcd for $\mathrm{C}_{26} \mathrm{H}_{22} \mathrm{ClF}_{2} \mathrm{NO}_{3} \mathrm{~S}: \mathrm{C}, 62.21$; H, 4.42; N, 2.79. Found: C, 62.27; H, 4.48; N, 2.72 .

Ethyl 5-[(4-Chlorophenyl)sulfanyl]-4-hydroxy-2,6-di(4-methylphenyl)1,2,5,6-tetrahydro-3-pyridinecarboxylate (2l): Isolated as colorless crystals $(0.250 \mathrm{~g}, 46 \%)$; mp $131-132{ }^{\circ} \mathrm{C} ;{ }^{1} \mathrm{H}-\mathrm{NMR}\left(300 \mathrm{MHz}, \mathrm{CDCl}_{3}\right) \delta_{\mathrm{H}}: 0.96(\mathrm{t}$, $\left.J=6.9 \mathrm{~Hz}, 3 \mathrm{H}, \mathrm{CH}_{3}\right), 2.29\left(\mathrm{~s}, 1 \mathrm{H}, \mathrm{CH}_{3}\right), 2.32\left(\mathrm{~s}, 1 \mathrm{H}, \mathrm{CH}_{3}\right), 3.67(\mathrm{~d}, J=9.9$ $\mathrm{Hz}, 1 \mathrm{H}, \mathrm{H}-6), 3.79$ (d, $J=9.9 \mathrm{~Hz}, 1 \mathrm{H}, \mathrm{H}-5), 3.85-4.03\left(\mathrm{~m}, 2 \mathrm{H}, \mathrm{CH}_{2}\right), 4.93$ $(\mathrm{s}, 1 \mathrm{H}, \mathrm{H}-2), 6.66-7.43\left(\mathrm{~m}, 12 \mathrm{H}\right.$, aromatic). ${ }^{13} \mathrm{C}-\mathrm{NMR}\left(75 \mathrm{MHz}, \mathrm{CDCl}_{3}\right)$ $\delta_{\mathrm{C}}: 14.3,21.5,21.6,53.9,55.8,56.4,59.5,97.1,127.6,128.3,128.9,129.5$, $29.7,135.7,136.3,137.0,138.3,139.0,141.5,155.1,169.1$. IR $(\mathrm{KBr}) v_{\max }$ 739, 1237, 1265, 1528, 1602, 1668, 2993, 3308, $3450 \mathrm{~cm}^{-1}$; Anal. Calcd for $\mathrm{C}_{28} \mathrm{H}_{28} \mathrm{ClNO}_{3} \mathrm{~S}$ : C, 68.07; H, 5.71; N, 2.84. Found: C, 68.13; H, 5.76; N, 2.79 .

Ethyl 5-[(4-Chlorophenyl)sulfanyl]-4-hydroxy-2,6-di(4-methylphenyl)1,2,5,6-tetrahydro-3-pyridinecarboxylate (31): Isolated as colorless crystals; $(0.033 \mathrm{~g}, 6 \%) ; \mathrm{mp} 120-122{ }^{\circ} \mathrm{C} ;{ }^{1} \mathrm{H}-\mathrm{NMR}\left(300 \mathrm{MHz}, \mathrm{CDCl}_{3}\right) \delta_{\mathrm{H}}: 0.94(\mathrm{t}$, $\left.J=7.2 \mathrm{~Hz}, 3 \mathrm{H}, \mathrm{CH}_{3}\right), 2.29\left(\mathrm{~s}, 1 \mathrm{H}, \mathrm{CH}_{3}\right), 2.30\left(\mathrm{~s}, 1 \mathrm{H}, \mathrm{CH}_{3}\right), 3.67(\mathrm{~s}, 1 \mathrm{H}, \mathrm{H}-5)$ $3.82-4.03\left(\mathrm{~m}, 1 \mathrm{H}, \mathrm{CH}_{2}\right), 4.57(\mathrm{~d}, 1 \mathrm{H}, J=1.8 \mathrm{~Hz}, \mathrm{H}-6), 4.97(\mathrm{~s}, 1 \mathrm{H}, \mathrm{H}-2)$,
6.67-7.41 (m, 12H, aromatic). ${ }^{13} \mathrm{C}-\mathrm{NMR}\left(75 \mathrm{MHz}, \mathrm{CDCl}_{3}\right) \delta_{\mathrm{C}}: 14.6,21.5$ 21.6, 58.4, 59.4, 59.6, 60.2, 96.5, 127.5, 128.4, 129.0, 129.3, 129.4, 129.7, $135.9,136.5,137.3,138.6,141.0,142.5,156.5,169.1$. IR (KBr) $v_{\text {ax }}: 742$, 1234, 1269, 1521, 1603, 1664, 2990, 3305, $3442 \mathrm{~cm}^{-1}$; Anal. Calcd for $\mathrm{C}_{28} \mathrm{H}_{28} \mathrm{ClNO}_{3} \mathrm{~S}$ : C, 68.07; H, 5.71; N, 2.84. Found: C, 68.12; H, 5.66; N, 2.88 .

Ethyl 2,6-Di(2-chlorophenyl)-5-[(4-chlorophenyl)sulfanyl]-4-hydroxy1,2,5,6-tetrahydro-3-pyridinecarboxylate $(\mathbf{3 m})$ : Isolated as colorless crystals; $(0.290 \mathrm{~g}, 49 \%) ; \mathrm{mp} 138-139{ }^{\circ} \mathrm{C} ;{ }^{1} \mathrm{H}-\mathrm{NMR}\left(300 \mathrm{MHz}, \mathrm{CDCl}_{3}\right) \delta_{\mathrm{H}}: 0.84(\mathrm{t}$, $\left.J=7.2 \mathrm{~Hz}, 3 \mathrm{H}, \mathrm{CH}_{3}\right), 1.94($ br s, $1 \mathrm{H}, \mathrm{NH}), 3.78-3.96\left(\mathrm{~m}, 2 \mathrm{H}, \mathrm{CH}_{2}\right), 3.97-$ $4.03(\mathrm{~m}, 1 \mathrm{H}, \mathrm{H}-5), 4.81$ (d, $J=1.5 \mathrm{~Hz}, 1 \mathrm{H}, \mathrm{H}-6), 5.55$ (s, 1H, H-2), 6.22 (brs, $1 \mathrm{H}, \mathrm{OH}), 6.87(\mathrm{~d}, J=8.4 \mathrm{~Hz}, 2 \mathrm{H}$, aromatic), $7.07(\mathrm{~d}, J=8.4 \mathrm{~Hz}, 2 \mathrm{H}$, aromatic), 7.15-7.54 (m, 8H, aromatic). ${ }^{13} \mathrm{C}-\mathrm{NMR}\left(75 \mathrm{MHz}, \mathrm{CDCl}_{3}\right) \delta_{\mathrm{C}}$ : $13.6,54.3,55.0,56.3,59.1,95.7,126.7,127.1,127.9,128.7,128.8,128.9$, $129.8,132.4,133.4,133.5,133.7,133.9,136.8,143.2,156.4,168.5$. IR (KBr) $v_{\max }: 740,1225,1261,1523,1614,1661,2986,3319,3445 \mathrm{~cm}^{-1}$; Anal. Calcd for $\mathrm{C}_{26} \mathrm{H}_{22} \mathrm{Cl}_{3} \mathrm{NO}_{3} \mathrm{~S}$ : C, 58.38; H, 4.15; N, 2.62. Found: C, 58.32; H, 4.22; N, 2.57 .

Ethyl 2,6-Di(2-bromophenyl)-5-[(4-chlorophenyl)sulfanyl]-4-hydroxy1,2,5,6-tetrahydro-3-pyridinecarboxylate (3n): Isolated as colorless solid; $(0.274 \mathrm{~g}, 40 \%)$; mp $121-122{ }^{\circ} \mathrm{C} ;{ }^{1} \mathrm{H}-\mathrm{NMR}\left(300 \mathrm{MHz}, \mathrm{CDCl}_{3}\right) \delta_{\mathrm{H}}: 0.90(\mathrm{t}$ $\left.J=7.2 \mathrm{~Hz}, 3 \mathrm{H}, \mathrm{CH}_{3}\right), 1.97$ (br s, $\left.1 \mathrm{H}, \mathrm{NH}\right), 3.91-4.05\left(\mathrm{~m}, 2 \mathrm{H}, \mathrm{CH}_{2}\right), 4.14(\mathrm{~s}$, 1H, H-5), 4.77 (s, 1H, H-6), 5.47 (s, 1H, H-2), 6.91-7.57 (m, 12H, aromatic), $12.3(\mathrm{~s}, 1 \mathrm{H}, \mathrm{OH}) .{ }^{13} \mathrm{C}-\mathrm{NMR}\left(75 \mathrm{MHz}, \mathrm{CDCl}_{3}\right) \delta_{\mathrm{C}}: 13.5,53.4,56.6$, $58.8,60.6,101.1,123.0,127.1,127.9,128.7,129.2,129.3,130.2,130.4$, $132.3,133.4,133.6,134.0,134.8,134.9,137.8,140.3,157.1,170.7$. IR (KBr) $v_{\max }: 741,1229,1260,1526,1619,1665,2985,3316,3442 \mathrm{~cm}^{-1}$; Anal. Calcd for $\mathrm{C}_{26} \mathrm{H}_{22} \mathrm{Br}_{2} \mathrm{ClNO}_{3} \mathrm{~S}$ : C, 50.06; H, 3.55; N, 2.25. Found C, 50.15; H, 3.49; N, 2.20.

Ethyl 5-[(4-Chlorophenyl)sulfanyl]-2,6-di(2,4-dichlorophenyl)-4-hydroxy1,2,5,6-tetrahydro-3-pyridinecarboxylate (3o): Isolated as pale yellow solid; $(0.332 \mathrm{~g}, 50 \%)$; mp $156-157{ }^{\circ} \mathrm{C} ;{ }^{1} \mathrm{H}-\mathrm{NMR}\left(300 \mathrm{MHz}, \mathrm{CDCl}_{3}\right) \delta_{\mathrm{C}}: 0.96(\mathrm{t}$, $\left.J=7.2 \mathrm{~Hz}, 3 \mathrm{H}, \mathrm{CH}_{3}\right), 1.87($ br s, $1 \mathrm{H}, \mathrm{NH}), 3.92-4.03\left(\mathrm{~m}, 2 \mathrm{H}, \mathrm{CH}_{2}\right), 4.06$ (s, $1 \mathrm{H}, \mathrm{H}-5), 4.75$ (s, 1H, H-6), $5.44(\mathrm{~s}, 1 \mathrm{H}, \mathrm{H}-2), 6.96-7.38(\mathrm{~m}, 9 \mathrm{H}$, aromatic), 7.47 (d, $J=8.4 \mathrm{~Hz}, 1 \mathrm{H}), 12.3(\mathrm{~s}, 1 \mathrm{H}, \mathrm{OH}) .{ }^{13} \mathrm{C}-\mathrm{NMR}(75 \mathrm{MHz}$, $\left.\mathrm{CDCl}_{3}\right) \delta_{\mathrm{C}}: 13.5,53.2,56.2,59.0,60.8,100.7,126.8,127.7,128.6,128.7$, $128.8,130.0,130.6,133.0,133.4,133.5,133.6,133.7,134.1,134.3,134.9$, $140.2,170.3,170.5$. IR (KBr) $v_{\max }: 739,1228,1260,1527,1615,1660$, 2984, 3318, $3447 \mathrm{~cm}^{-1}$; Anal. Calcd for $\mathrm{C}_{26} \mathrm{H}_{20} \mathrm{Cl}_{5} \mathrm{NO}_{3} \mathrm{~S}: \mathrm{C}, 51.72 ; \mathrm{H}, 3.34$; N, 2.32. Found: C, 51.80; H, 3.25; N, 2.26.

Ethyl 5-[(4-Chlorophenyl)sulfanyl]-4-hydroxy-2,6-di(3-nitrophenyl)1,2,5,6-tetrahydro-3-pyridinecarboxylate (2p and $\mathbf{3 p}$ ) (Appears as Mixture in a Ratio $1: 0.88)$ : Isolated as viscous liquid; $(0.293 \mathrm{~g}, 48 \%) ;{ }^{1} \mathrm{H}-\mathrm{NMR}(300$ $\left.\mathrm{MHz}, \mathrm{CDCl}_{3}\right) \delta_{\mathrm{H}}: 0.84-1.05\left(\mathrm{~m}, \mathrm{CH}_{3}\right), 3.82-4.16\left(\mathrm{~m}, \mathrm{CH}_{2}\right), 4.56-4.65$ (m, H-5), 4.80-5.15 (m, H-6, H-2), 7.08-8.27 (m, aromatic), $12.6(\mathrm{~s}, \mathrm{OH})$, 12.9 (s, OH). HR-MS (ESI) $\mathrm{m} / z$ : Calcd for $\mathrm{C}_{26} \mathrm{H}_{22}{ }^{35} \mathrm{ClN}_{3} \mathrm{O}_{7} \mathrm{~S}: 555.0867$; Found, $554.3712(\mathrm{M}-1)$.

Ethyl 4-Hydroxy-5-[(4-methylphenyl)sulfanyl]-2,6-diphenyl-1,2,5,6tetrahydro-3-pyridinecarboxylate $(\mathbf{2 q})$ : Isolated as colorless crystals; $(0.244$ g, 46\%); mp $110-111^{\circ} \mathrm{C}$; ${ }^{1} \mathrm{H}-\mathrm{NMR}\left(300 \mathrm{MHz}, \mathrm{CDCl}_{3}\right) \delta_{\mathrm{H}}: 0.92(\mathrm{t}$, $\left.J=7.2 \mathrm{~Hz}, 3 \mathrm{H}, \mathrm{CH}_{3}\right), 2.41\left(\mathrm{~s}, 1 \mathrm{H}, \mathrm{CH}_{3}\right), 3.78$ (s, 2H, H-5, H-6), 3.87-4.01 $\left(\mathrm{m}, 2 \mathrm{H}, \mathrm{CH}_{2}\right), 4.95(\mathrm{~s}, 1 \mathrm{H}, \mathrm{H}-2), 6.78-7.29$ (m, 14H, aromatic). ${ }^{13} \mathrm{C}-\mathrm{NMR}$ $\left(75 \mathrm{MHz} \mathrm{CDCl}_{3}\right) \delta_{\mathrm{C}}: 14.5,21.7,53.8,56.0,57.0,59.4,96.4,126.7,127.1$, $127.8,128.1,128.4,128.5,128.9,130.4,136.0,139.6,142.2,144.6,155.8$, 169.0. IR (KBr) $v_{\mathrm{max}}: 735,1234,1261,1525,1605,1662,2990,3311,3442$ $\mathrm{cm}^{-1}$; Anal. Calcd for $\mathrm{C}_{27} \mathrm{H}_{27} \mathrm{NO}_{3} \mathrm{~S}$ : C, 72.78; H, 6.11; N, 3.14. Found: C, $72.84 ; \mathrm{H}, 6.17 ; \mathrm{N}, 3.21$.

Ethyl 4-Hydroxy-5-[(4-methylphenyl)sulfanyl]-2,6-diphenyl-1,2,5,6-tetrahydro-3-pyridinecarboxylate (3q): Isolated as colorless crystals; $(0.042 \mathrm{~g}, 8 \%)$; mp $107-108^{\circ} \mathrm{C} ;{ }^{1} \mathrm{H}-\mathrm{NMR}\left(300 \mathrm{MHz}, \mathrm{CDCl}_{3}\right) \delta_{\mathrm{H}}: 0.74(\mathrm{t}, J=7.2 \mathrm{~Hz}, 3 \mathrm{H}$, $\left.\mathrm{CH}_{3}\right), 1.79$ (br s, $\left.1 \mathrm{H}, \mathrm{NH}\right), 3.45$ (s, $\left.1 \mathrm{H}, \mathrm{H}-5\right), 3.69-4.02\left(\mathrm{~m}, 2 \mathrm{H}, \mathrm{CH}_{2}\right), 4.40$ (d, $J=1.5 \mathrm{~Hz}, 1 \mathrm{H}, \mathrm{H}-6), 4.87$ (s, $1 \mathrm{H}, \mathrm{H}-2), 6.00$ (br s, $1 \mathrm{H}, \mathrm{OH}), 6.72-7.41$ (m, 14H, aromatic). ${ }^{13} \mathrm{C}-\mathrm{NMR}\left(75 \mathrm{MHz}, \mathrm{CDCl}_{3}\right) \delta_{\mathrm{C}}: 13.7,58.3,58.9,59.1$, $59.5,96.0,126.8,127.3,127.9,128.0,128.1,128.3,129.6,131.9,135.5$, $137.9,139.8,145.9,157.1,168.9$. IR $(\mathrm{KBr}) v_{\max }: 738,1231,1265,1529$, 1601, 1659, 2993, 3315, $3440 \mathrm{~cm}^{-1}$; Anal. Calcd for $\mathrm{C}_{27} \mathrm{H}_{27} \mathrm{NO}_{3} \mathrm{~S}: \mathrm{C}, 72.78$ H, 6.11; N, 3.14. Found: C, 72.82; H, 6.07; N, 3.20.

Ethyl 2,6-Di(4-chlorophenyl)-4-hydroxy-5-[(4-methylphenyl)sulfanyl]1,2,5,6-tetrahydro-3-pyridinecarboxylate (2r): Isolated as colorless crystals; $(0.306 \mathrm{~g}, 50 \%) ; \mathrm{mp} 148-149^{\circ} \mathrm{C} ;{ }^{1} \mathrm{H}-\mathrm{NMR}\left(300 \mathrm{MHz}, \mathrm{CDCl}_{3}\right) \delta_{\mathrm{H}}: 0.95(\mathrm{t}$, $\left.J=7.2 \mathrm{~Hz}, 3 \mathrm{H}, \mathrm{CH}_{3}\right), 2.43\left(\mathrm{~s}, 1 \mathrm{H}, \mathrm{CH}_{3}\right), 3.68$ (s, 2H, H-5, H-6), 3.89-4.02 $\left(\mathrm{m}, 2 \mathrm{H}, \mathrm{CH}_{2}\right), 4.90(\mathrm{~s}, 1 \mathrm{H}, \mathrm{H}-2), 6.71-7.33\left(\mathrm{~m}, 12 \mathrm{H}\right.$, aromatic). ${ }^{13} \mathrm{C}-\mathrm{NMR}$ $\left(75 \mathrm{MHz}, \mathrm{CDCl}_{3}\right) \delta_{\mathrm{C}}: 14.5,21.7,53.4,55.4,56.2,59.6,96.2,126.7,128.3$, $129.1,129.2,129.8,130.5,132.4,134.2,136.0,139.9,140.5,143.2,155.7$, 
168.8. IR (KBr) $v_{\max }: 739,1230,1267,1525,1601,1660,2987,3308,3445$ $\mathrm{cm}^{-1}$; Anal. Calcd for $\mathrm{C}_{27} \mathrm{H}_{25} \mathrm{Cl}_{2} \mathrm{NO}_{3} \mathrm{~S}: \mathrm{C}, 63.03 ; \mathrm{H}, 4.90 ; \mathrm{N}, 2.72$. Found: $\mathrm{C}$, 63.09; H, 4.84; N, 2.67.

Ethyl 2,6-Di(4-chlorophenyl)-4-hydroxy-5-[(4-methylphenyl)sulfanyl]1,2,5,6-tetrahydro-3-pyridinecarboxylate (3r): Isolated as colorless crystals; $(0.043 \mathrm{~g}, 7 \%) ; \mathrm{mp} 144-146^{\circ} \mathrm{C} ;{ }^{1} \mathrm{H}-\mathrm{NMR}\left(300 \mathrm{MHz}, \mathrm{CDCl}_{3}\right) \delta_{\mathrm{H}}: 0.89$ (t, $\left.J=7.2 \mathrm{~Hz}, 3 \mathrm{H}, \mathrm{CH}_{3}\right), 1.79$ (br s, $\left.1 \mathrm{H}, \mathrm{NH}\right), 2.29\left(\mathrm{~s}, 1 \mathrm{H}, \mathrm{CH}_{3}\right), 3.53$ (dd, $1 \mathrm{H}$, $J=2.1,1.2 \mathrm{~Hz}, \mathrm{H}-5), 3.84-3.95\left(\mathrm{~m}, 2 \mathrm{H}, \mathrm{CH}_{2}\right), 4.45(\mathrm{~d}, J=2.1 \mathrm{~Hz}, 1 \mathrm{H}, \mathrm{H}-6)$, 4.92 (s, $1 \mathrm{H}, \mathrm{H}-2), 6.12$ (br s, $1 \mathrm{H}, \mathrm{OH}), 6.85-7.33$ (m, 12H, aromatic) ${ }^{13} \mathrm{C}-$ NMR $\left(75 \mathrm{MHz}, \mathrm{CDCl}_{3}\right) \delta_{\mathrm{C}}: 14.3,21.5,58.2,59.0,59.2,59.5,95.9,128.6$, $129.1,129.8,130.3,131.9,132.9,133.6,133.7,138.6,138.7,140.4,144.9$, 157.3, 169.1. IR (KBr) $v_{\max }: 738,1235,1262,1524,1606,1665,2991$, 3312, $3441 \mathrm{~cm}^{-1}$; Anal. Calcd for $\mathrm{C}_{27} \mathrm{H}_{25} \mathrm{Cl}_{2} \mathrm{NO}_{3} \mathrm{~S}$ : C, 63.03; H, 4.90; N, 2.72. Found: C, 63.10; H, 4.95; N, 2.76 .

Ethyl 2,6-Di(4-fluorophenyl)-4-hydroxy-5-[(4-methylphenyl)sulfanyl]1,2,5,6-tetrahydro-3-pyridinecarboxylate (2s): Isolated as colorless crystals; $(0.160 \mathrm{~g}, 28 \%)$; mp $112-113{ }^{\circ} \mathrm{C} ;{ }^{1} \mathrm{H}-\mathrm{NMR}\left(300 \mathrm{MHz}, \mathrm{CDCl}_{3}\right) \delta_{\mathrm{H}}: 0.94(\mathrm{t}$, $\left.J=7.2 \mathrm{~Hz}, 3 \mathrm{H}, \mathrm{CH}_{3}\right), 2.43\left(\mathrm{~s}, 1 \mathrm{H}, \mathrm{CH}_{3}\right), 3.70(\mathrm{~s}, 2 \mathrm{H}, \mathrm{H}-5, \mathrm{H}-6), 3.86-4.03$ $\left(\mathrm{m}, 2 \mathrm{H}, \mathrm{CH}_{2}\right), 4.92(\mathrm{~s}, 1 \mathrm{H}, \mathrm{H}-2), 6.68-7.19\left(\mathrm{~m}, 12 \mathrm{H}\right.$, aromatic). ${ }^{13} \mathrm{C}-\mathrm{NMR}$ $\left(75 \mathrm{MHz}, \mathrm{CDCl}_{3}\right) \delta_{\mathrm{C}}: 14.5,21.7,53.7,55.3,56.2,59.5,96.4,114.7,115.0$, $115.7,116.0,126.8,129.2,129.3,130.0,130.1,130.4,136.0,137.8,137.9$, $139.8,140.4,140.5,160.2,161.1,163.4,164.4,155.6,168.8 . \mathrm{IR}(\mathrm{KBr}) v_{\max }$ : 738, 1230, 1264, 1530, 1609, 1662, 2997, 3306, $3440 \mathrm{~cm}^{-1}$; Anal. Calcd for $\mathrm{C}_{27} \mathrm{H}_{25} \mathrm{~F}_{2} \mathrm{NO}_{3} \mathrm{~S}$ : C, 67.34; H, 5.23; N, 2.91. Found: C, 67.30; H, 5.29; N, 2.85 .

Ethyl 2,6-Di(4-fluorophenyl)-4-hydroxy-5-[(4-methylphenyl)sulfanyl]1,2,5,6-tetrahydro-3-pyridinecarboxylate (3s): Isolated as colorless crystals; $(0.150 \mathrm{~g}, 26 \%)$; mp $110-111^{\circ} \mathrm{C}$; ${ }^{1} \mathrm{H}-\mathrm{NMR}\left(300 \mathrm{MHz}, \mathrm{CDCl}_{3}\right) \delta_{\mathrm{H}}: 0.87(\mathrm{t}$, $\left.J=7.2 \mathrm{~Hz}, 3 \mathrm{H}, \mathrm{CH}_{3}\right), 1.75$ (br s, $\left.1 \mathrm{H}, \mathrm{NH}\right), 2.27\left(\mathrm{~s}, 1 \mathrm{H}, \mathrm{CH}_{3}\right), 3.56(\mathrm{dd}, 1 \mathrm{H}$ $J=2.1,1.2 \mathrm{~Hz}, \mathrm{H}-5), 3.78-3.99\left(\mathrm{~m}, 2 \mathrm{H}, \mathrm{CH}_{2}\right), 4.45(\mathrm{~d}, J=2.1 \mathrm{~Hz}, 1 \mathrm{H}, \mathrm{H}-6)$, 4.94 (s, 1H, H-2), 6.09 (brs, $1 \mathrm{H}, \mathrm{OH}), 6.84-7.34$ (m, 12H, aromatic). ${ }^{13} \mathrm{C}-$ NMR $\left(75 \mathrm{MHz}, \mathrm{CDCl}_{3}\right) \delta_{\mathrm{C}}: 14.3,21.5,58.6,59.0,59.2,59.4,96.2,115.1$, $115.2,115.3,115.4,129.3,129.4,129.9,130.0,130.2,132.1,133.6,135.9$, $136.0,138.6,142.1,142.2,160.6,160.9,163.9,164.2,157.4,169.2$. IR $(\mathrm{KBr}) v_{\max }: 735,1236,1268,1532,1603,1669,2991,3310,3447 \mathrm{~cm}^{-1}$; Anal. Calcd for $\mathrm{C}_{27} \mathrm{H}_{25} \mathrm{~F}_{2} \mathrm{NO}_{3} \mathrm{~S}$ : C, 67.34; H, 5.23; N, 2.91. Found: C, 67.39; H, 5.18; N, 2.97 .

Ethyl 4-Hydroxy-2,6-di(4-methylphenyl)-5-[(4-methylphenyl)sulfanyl]1,2,5,6-tetrahydro-3-pyridinecarboxylate (2t): Isolated as colorless crystals; $(0.282 \mathrm{~g}, 50 \%) ; \mathrm{mp} 137-138^{\circ} \mathrm{C}$; ${ }^{1} \mathrm{H}-\mathrm{NMR}\left(300 \mathrm{MHz}, \mathrm{CDCl}_{3}\right) \delta_{\mathrm{H}}: 0.95(\mathrm{t}$, $\left.J=7.2 \mathrm{~Hz}, 3 \mathrm{H}, \mathrm{CH}_{3}\right), 2.27\left(\mathrm{~s}, 1 \mathrm{H}, \mathrm{CH}_{3}\right), 2.33\left(\mathrm{~s}, 1 \mathrm{H}, \mathrm{CH}_{3}\right), 2.42(\mathrm{~s}, 1 \mathrm{H}$, $\left.\mathrm{CH}_{3}\right), 3.72(\mathrm{~d}, J=10.0 \mathrm{~Hz}, 1 \mathrm{H}, \mathrm{H}-5), 3.76(\mathrm{~d}, J=10.0 \mathrm{~Hz}, 1 \mathrm{H}, \mathrm{H}-6), 3.74$ $4.03\left(\mathrm{~m}, 2 \mathrm{H}, \mathrm{CH}_{2}\right), 4.91(\mathrm{~s}, 1 \mathrm{H}, \mathrm{H}-2), 6.64-7.37\left(\mathrm{~m}, 12 \mathrm{H}\right.$, aromatic) ${ }^{13} \mathrm{C}-$ NMR $\left(75 \mathrm{MHz}, \mathrm{CDCl}_{3}\right) \delta_{\mathrm{C}}: 14.6,21.5,21.6,21.7,53.8,55.6,56.6,59.4$, $96.6,127.1,127.8,128.4,128.8,129.6,130.4,135.9,136.0,138.1,139.3$, $139.5,141.7,155.6,168.8$. IR (KBr) $v_{\max }: 741,1235,1261,1534,1607$, 1665, 2993, 3312, 3441 $\mathrm{cm}^{-1}$; Anal. Calcd for $\mathrm{C}_{29} \mathrm{H}_{31} \mathrm{NO}_{3} \mathrm{~S}: \mathrm{C}, 73.54 ; \mathrm{H}$, 6.60 ; N, 2.96. Found: C, 73.50; H, 6.67; N, 2.91 .

Ethyl 4-Hydroxy-2,6-di(4-methylphenyl)-5-[(4-methylphenyl)sulfanyl]1,2,5,6-tetrahydro-3-pyridinecarboxylate (3t): Isolated as colorless crystals; $(0.045 \mathrm{~g}, 8 \%) ; \mathrm{mp} 120-120^{\circ} \mathrm{C} ;{ }^{1} \mathrm{H}-\mathrm{NMR}\left(300 \mathrm{MHz}, \mathrm{CDCl}_{3}\right) \delta_{\mathrm{H}}: 0.84(\mathrm{t}$, $\left.J=7.2 \mathrm{~Hz}, 3 \mathrm{H}, \mathrm{CH}_{3}\right), 1.79$ (br s, $\left.1 \mathrm{H}, \mathrm{NH}\right), 2.25\left(\mathrm{~s}, 1 \mathrm{H}, \mathrm{CH}_{3}\right), 2.32(\mathrm{~s}, 1 \mathrm{H}$, $\left.\mathrm{CH}_{3}\right), 2.45\left(\mathrm{~s}, 1 \mathrm{H}, \mathrm{CH}_{3}\right), 3.54(\mathrm{dd}, 1 \mathrm{H}, J=2.0,1.2 \mathrm{~Hz}, \mathrm{H}-5), 3.77-4.00(\mathrm{~m}$, $\left.2 \mathrm{H}, \mathrm{CH}_{2}\right), 4.43$ (d, J=2.0 Hz, 1H, H-6), $4.90(\mathrm{~s}, 1 \mathrm{H}, \mathrm{H}-2), 6.04$ (br s, $1 \mathrm{H}$, $\mathrm{OH}), 6.75-7.43\left(\mathrm{~m}, 12 \mathrm{H}\right.$, aromatic). ${ }^{13} \mathrm{C}-\mathrm{NMR}\left(75 \mathrm{MHz}, \mathrm{CDCl}_{3}\right) \delta_{\mathrm{C}}: 14.2$, 21.3, 21.6, 21.9, 58.5, 58.9, 59.0, 59.1, 96.3, 127.5, 127.9, 128.3, 128.5, $129.7,130.2,135.7,136.5,138.6,139.1,139.2,141.8,157.2,169.5$. IR (KBr) $v_{\max }: 737,1230,1259,1538,1602,1662,2984,3305,3452 \mathrm{~cm}^{-1}$; Anal. Calcd for $\mathrm{C}_{29} \mathrm{H}_{31} \mathrm{NO}_{3} \mathrm{~S}$ : C, 73.54; H, 6.60; N, 2.96. Found: C, 73.59; $\mathrm{H}, 6.56 ; \mathrm{N}, 2.92$.

Ethyl 2,6-Di(2-chlorophenyl)-4-hydroxy-5-[(4-methylphenyl)sulfanyl]1,2,5,6-tetrahydro-3-pyridinecarboxylate (3u): Isolated as colorless crystals; $(0.305 \mathrm{~g}, 50 \%)$; mp $133-134{ }^{\circ} \mathrm{C}$; ${ }^{1} \mathrm{H}-\mathrm{NMR}\left(300 \mathrm{MHz}, \mathrm{CDCl}_{3}\right) \delta_{\mathrm{H}}: 0.83(\mathrm{t}$, $\left.J=7.2 \mathrm{~Hz}, 3 \mathrm{H}, \mathrm{CH}_{3}\right), 1.88(\mathrm{brs}, 1 \mathrm{H}, \mathrm{NH}), 2.25\left(\mathrm{~s}, 1 \mathrm{H}, \mathrm{CH}_{3}\right), 3.70-4.20(\mathrm{~m}$, $3 \mathrm{H}, \mathrm{CH}_{2}$ and $\left.\mathrm{H}-5\right), 4.70-4.89$ (m, 1H, H-6), 5.54 (s, 1H, H-2), 6.19 (brs, $1 \mathrm{H}, \mathrm{OH}), 6.83(\mathrm{~d}, J=7.8 \mathrm{~Hz}, 2 \mathrm{H}$, aromatic), $6.92(\mathrm{~d}, J=7.8 \mathrm{~Hz}, 2 \mathrm{H}$, aromatic), $7.10-7.55\left(\mathrm{~m}, 8 \mathrm{H}\right.$, aromatic). ${ }^{13} \mathrm{C}-\mathrm{NMR}\left(75 \mathrm{MHz}, \mathrm{CDCl}_{3}\right) \delta_{\mathrm{C}}: 13.6$, 21.0, 54.6, 55.0, 56.4, 59.0, 95.0, 126.5, 127.1, 127.8, 128.6, 128.8, 128.9, $129.4,129.7,130.0,131.9,132.4,132.7,133.7,137.1,137.7,143.5,157.5$, 168.6. IR (KBr) $v_{\max }: 746,1227,1261,1521,1610,1664,2985,3315$, $3442 \mathrm{~cm}^{-1}$; Anal. Calcd for $\mathrm{C}_{27} \mathrm{H}_{25} \mathrm{Cl}_{2} \mathrm{NO}_{3} \mathrm{~S}$ : C, 63.03; H, 4.90; N, 2.72. Found: C, 63.08; H, 4.97; N, 2.65.

Ethyl 2,6-Di(2,4-dichlorophenyl)-4-hydroxy-5-[(4-methylphenyl)sulfanyl]1,2,5,6-tetrahydro-3-pyridinecarboxylate (3v): Isolated as pale yellow solid;
(0.312 g, 45\%); mp 141-142 ${ }^{\circ} \mathrm{C} ;{ }^{1} \mathrm{H}-\mathrm{NMR}\left(300 \mathrm{MHz}, \mathrm{CDCl}_{3}\right) \delta_{\mathrm{H}}: 0.95(\mathrm{t}$, $\left.J=7.2 \mathrm{~Hz}, 3 \mathrm{H}, \mathrm{CH}_{3}\right), 1.87$ (br s, $\left.1 \mathrm{H}, \mathrm{NH}\right), 2.27\left(\mathrm{~s}, 3 \mathrm{H}, \mathrm{CH}_{3}\right), 3.94-4.03(\mathrm{~m}$, $\left.2 \mathrm{H}, \mathrm{CH}_{2}\right), 4.06(\mathrm{~s}, 1 \mathrm{H}, \mathrm{H}-5), 4.72(\mathrm{~s}, 1 \mathrm{H}, \mathrm{H}-6), 5.43(\mathrm{~s}, 1 \mathrm{H}, \mathrm{H}-2), 6.89-$ $6.95(\mathrm{~m}, 4 \mathrm{H}$, aromatic), $7.15(\mathrm{~d}, J=2.1 \mathrm{~Hz}, 1 \mathrm{H}), 7.22-7.29$ (m, 3H, aromatic), 7.38 (d, $J=1.8 \mathrm{~Hz}, 1 \mathrm{H}), 7.47(\mathrm{~d}, J=8.4 \mathrm{~Hz}, 1 \mathrm{H}), 12.3$ (s, 1H, OH). ${ }^{13} \mathrm{C}-\mathrm{NMR}\left(75 \mathrm{MHz}, \mathrm{CDCl}_{3}\right) \delta_{\mathrm{C}}: 13.5,21.1,53.1,56.2,59.0,60.7,100.5$, $126.7,127.7,128.5,128.7,129.0,129.5,130.7,131.2,132.5,133.0,133.3$, $134.0,134.4,135.0,137.7,140.3,170.5,170.9$. IR (KBr) $v_{\max }: 743,1228$, $1265,1520,1614,1667,2988,3318,3446 \mathrm{~cm}^{-1}$; Anal. Calcd for $\mathrm{C}_{27} \mathrm{H}_{23} \mathrm{Cl}_{4} \mathrm{NO}_{3} \mathrm{~S}: \mathrm{C}, 55.59 ; \mathrm{H}, 3.97 ; \mathrm{N}, 2.40$. Found: C, 55.55; H, 3.91; N, 2.48 .

Ethyl 4-Hydroxy-5-[(4-methylphenyl)sulfanyl]-2,6-di(3-nitrophenyl)1,2,5,6-tetrahydro-3-pyridinecarboxylate (2w and 3w) (Appears as Mixture in a Ratio 1:0.30): Isolated as viscous liquid; $(0.293 \mathrm{~g}, 46 \%) ;{ }^{1} \mathrm{H}-\mathrm{NMR}$ $\left(300 \mathrm{MHz}, \mathrm{CDCl}_{3}\right) \delta_{\mathrm{H}}: 0.83-1.01\left(\mathrm{~m}, \mathrm{CH}_{3}\right), 2.23\left(\mathrm{~s}, \mathrm{CH}_{3}\right), 2.27\left(\mathrm{~s}, \mathrm{CH}_{3}\right)$, $3.86-4.21\left(\mathrm{~m}, \mathrm{CH}_{2}\right), 4.58-5.10(\mathrm{~m}, \mathrm{H}-5, \mathrm{H}-6, \mathrm{H}-2), 6.91-8.48$ (m, aromatic), $12.3(\mathrm{~s}, \mathrm{OH}), 12.8(\mathrm{~s}, \mathrm{OH})$. HR-MS (ESI) $\mathrm{m} / \mathrm{z}$ : Calcd for $\mathrm{C}_{27} \mathrm{H}_{25} \mathrm{~N}_{3} \mathrm{O}_{7} \mathrm{~S}$ : 535.1413; Found, 534.2901 (M-1).

Synthesis of Ethyl 4-Hydroxy-2,6-diaryl-5-(arylsulfanyl)-3-pyridinecarboxylates (5). General Procedure To a solution of ethyl 4-hydroxy-2,6diaryl-5-(arylsulfanyl)-1,2,5,6-tetrahydro-3-pyridinecarboxylate $(1 \mathrm{mmol})$ in benzene $(20 \mathrm{ml})$, dichloro-dicyanobenzoquinone $(2 \mathrm{mmol})$ was added and refluxed over a water bath for $30 \mathrm{~min}$. The precipitated DDQ- $\mathrm{H}_{2}$ was filtered off and the filterate evaporated under vaccum. The residue was crystallized from ethanol to give the product.

Ethyl 4-Hydroxy-2,6-diphenyl-5-(phenylsulfanyl)-3-pyridinecarboxylate (5a): Isolated as colorless crystals $(0.072 \mathrm{~g}, 73 \%)$; mp $148-149{ }^{\circ} \mathrm{C} ;{ }^{1} \mathrm{H}-$ NMR $\left(300 \mathrm{MHz}, \mathrm{CDCl}_{3}\right) \delta_{\mathrm{H}}: 0.78\left(\mathrm{t}, J=7.2 \mathrm{~Hz}, 3 \mathrm{H}, \mathrm{CH}_{3}\right), 3.98$ (q, $J=$ $\left.7.2 \mathrm{~Hz}, 2 \mathrm{H}, \mathrm{CH}_{2}\right), 6.57$ (br s, $\left.1 \mathrm{H}, \mathrm{OH}\right), 7.06-7.57$ (m, $15 \mathrm{H}$, aromatic). ${ }^{13} \mathrm{C}-$ NMR $\left(75 \mathrm{MHz}, \mathrm{CDCl}_{3}\right) \delta_{\mathrm{C}}: 13.6,61.5,108.2,108.7,126.2,126.5,128.0$, $128.4,128.8,128.9,129.0,129.6,129.7,135.8,140.8,142.3,156.5,161.5$, 165.2 , 169.6. IR (KBr) $v_{\max }: 732,1020,1099,1245,1480,1536,1585$, 1679, 2971, $3355 \mathrm{~cm}^{-1}$; Anal. Calcd for $\mathrm{C}_{26} \mathrm{H}_{21} \mathrm{NO}_{3} \mathrm{~S}$ : C, 73.04; H, 4.95; N, 3.28. Found: C, 73.10; H, 4.99; N, 3.22.

Ethyl 2,6-Di(4-chlorophenyl)-4-hydroxy-5-(phenylsulfanyl)-3-pyridinecarboxylate $(\mathbf{5 b})$ : Isolated as colorless crystals $(0.083 \mathrm{~g}, 84 \%)$; mp $115-$ $116^{\circ} \mathrm{C}$; ${ }^{1} \mathrm{H}-\mathrm{NMR}\left(300 \mathrm{MHz}, \mathrm{CDCl}_{3}\right) \delta_{\mathrm{H}}: 0.87\left(\mathrm{t}, J=7.2 \mathrm{~Hz}, 3 \mathrm{H}, \mathrm{CH}_{3}\right), 4.02$ $\left(\mathrm{q}, J=7.2 \mathrm{~Hz}, 2 \mathrm{H}, \mathrm{CH}_{2}\right), 6.65($ br s, $1 \mathrm{H}, \mathrm{OH}), 6.65-7.50(\mathrm{~m}, 13 \mathrm{H}$, aromatic). ${ }^{13} \mathrm{C}-\mathrm{NMR}\left(75 \mathrm{MHz}, \mathrm{CDCl}_{3}\right) \delta_{\mathrm{C}}: 13.8,61.6,108.0,109.0,126.5$, $128.2,128.6,129.8,130.2,131.0,135.1,135.2,135.3,139.0,140.6,142.3$, $156.7,160.4,164.0,169.2$. IR (KBr) $v_{\max }: 738,1012,1083,1238,1486$, 1525, 1587, 1683, 2985, $3336 \mathrm{~cm}^{-1}$; Anal. Calcd for $\mathrm{C}_{26} \mathrm{H}_{19} \mathrm{Cl}_{2} \mathrm{NO}_{3} \mathrm{~S}: \mathrm{C}$, $62.91 ; \mathrm{H}, 3.86 ; \mathrm{N}, 2.82$. Found: C, $62.85 ; \mathrm{H}, 3.80 ; \mathrm{N}, 2.87$.

Ethyl 2,6-Di(4-fluorophenyl)-4-hydroxy-5-(phenylsulfanyl)-3-pyridinecarboxylate (5c): Isolated as colorless crystals; $(0.074 \mathrm{~g}, 75 \%) ; \mathrm{mp} 117-$ $118^{\circ} \mathrm{C}$; ${ }^{1} \mathrm{H}-\mathrm{NMR}\left(300 \mathrm{MHz}, \mathrm{CDCl}_{3}\right) \delta_{\mathrm{H}}: 0.87\left(\mathrm{t}, J=7.2 \mathrm{~Hz}, 3 \mathrm{H}, \mathrm{CH}_{3}\right), 4.02$ (q, $\left.J=7.2 \mathrm{~Hz}, 2 \mathrm{H}, \mathrm{CH}_{2}\right), 6.62($ br s, $1 \mathrm{H}, \mathrm{OH}), 6.62-7.56(\mathrm{~m}, 13 \mathrm{H}$, aromatic). ${ }^{13} \mathrm{C}-\mathrm{NMR}\left(75 \mathrm{MHz}, \mathrm{CDCl}_{3}\right) \delta_{\mathrm{C}}: 13.7,61.6,108.1,108.8,114.9$, $115.1,115.2,115.5,126.4,126.5,129.8,130.7,130.8,131.5,131.6,135.5$, 136.6, 138.3, 156.7, 160.3, 161.8, 161.9, 164.1, 165.1, 165.2, 169.4. IR $(\mathrm{KBr}) v_{\max }: 735,1015,1080,1239,1480,1533,1592,1681,2989,3339$ $\mathrm{cm}^{-1}$; Anal. Calcd for $\mathrm{C}_{26} \mathrm{H}_{19} \mathrm{~F}_{2} \mathrm{NO}_{3} \mathrm{~S}$ : C, 67.37; H, 4.13; N, 3.02. Found: $\mathrm{C}$, 67.41; H, 4.08; N, 3.09.

Ethyl 4-Hydroxy-2,6-di(4-methylphenyl)-5-(phenylsulfanyl)-3-pyridinecarboxylate (5d): Isolated as colorless crystals; $(0.071 \mathrm{~g}, 72 \%) ; \mathrm{mp} 107-$ $109^{\circ} \mathrm{C} ;{ }^{1} \mathrm{H}-\mathrm{NMR}\left(300 \mathrm{MHz}, \mathrm{CDCl}_{3}\right) \delta_{\mathrm{H}}: 0.80\left(\mathrm{t}, J=6.6 \mathrm{~Hz}, 3 \mathrm{H}, \mathrm{CH}_{3}\right), 2.31$ (s, $\left.1 \mathrm{H}, \mathrm{CH}_{3}\right), 2.35\left(\mathrm{~s}, 1 \mathrm{H}, \mathrm{CH}_{3}\right), 3.85\left(\mathrm{q}, J=6.6 \mathrm{~Hz}, 2 \mathrm{H}, \mathrm{CH}_{2}\right), 6.60(\mathrm{br} \mathrm{s}, 1 \mathrm{H}$, $\mathrm{OH}), 6.62-7.56\left(\mathrm{~m}, 13 \mathrm{H}\right.$, aromatic). ${ }^{13} \mathrm{C}-\mathrm{NMR}\left(75 \mathrm{MHz}, \mathrm{CDCl}_{3}\right) \delta_{\mathrm{C}}: 13.6$, $21.7,21.8,61.5,108.2,108.6,126.3,126.5,128.7,128.9,129.0,129.5$, $129.7,135.7,139.0,156.6,161.1,164.8,169.5$. IR (KBr) $v_{\max }: 739,1017$, 1086, 1237, 1482,1538, 1590, 1686, 2992, $3341 \mathrm{~cm}^{-1}$; Anal. Calcd for $\mathrm{C}_{28} \mathrm{H}_{25} \mathrm{NO}_{3} \mathrm{~S}$ : C, 73.82; H, 5.53; N, 3.07. Found: C, 73.87; H, 5.59; N, 3.00.

Ethyl 2,6-Di(2-chlorophenyl)-4-hydroxy-5-(phenylsulfanyl)-3-pyridinecarboxylate (5e): Isolated as colorless crystals; $(0.076 \mathrm{~g}, 77 \%)$; mp $135-$ $136^{\circ} \mathrm{C}$; ${ }^{1} \mathrm{H}-\mathrm{NMR}\left(300 \mathrm{MHz}, \mathrm{CDCl}_{3}\right) \delta_{\mathrm{H}}: 0.79\left(\mathrm{t}, J=7.2 \mathrm{~Hz}, 3 \mathrm{H}, \mathrm{CH}_{3}\right)$, $3.92-4.31\left(\mathrm{~m}, 2 \mathrm{H}, \mathrm{CH}_{2}\right), 6.62-7.56\left(\mathrm{~m}, 13 \mathrm{H}\right.$, aromatic). ${ }^{13} \mathrm{C}-\mathrm{NMR}(75$ $\left.\mathrm{MHz}, \mathrm{CDCl}_{3}\right) \delta_{\mathrm{C}}: 13.5,61.5,108.9,112.2,126.6,127.2,127.3,129.0$, $129.3,129.4,129.6,129.7,129.9,130.7,132.2,132.8,133.1,134.7,139.6$, $141.9,156.7,159.6,163.9,168.3$. IR (KBr) $v_{\max }: 734,1013,1083,1240$, 1479, 1531, 1593, 1682, 2990, $3347 \mathrm{~cm}^{-1}$; Anal. Calcd for $\mathrm{C}_{26} \mathrm{H}_{19} \mathrm{Cl}_{2} \mathrm{NO}_{3} \mathrm{~S}$ : C, 62.91; H, 3.86; N, 2.82. Found: C, 62.95; H, 3.80; N, 2.75 .

Ethyl 2,6-Di(2-bromophenyl)-4-hydroxy-5-(phenylsulfanyl)-3-pyridinecarboxylate (5f): Isolated as solid $(0.071 \mathrm{~g}, 72 \%)$; m 103-104 ${ }^{\circ} \mathrm{C} ;{ }^{1} \mathrm{H}-\mathrm{NMR}$ $\left(300 \mathrm{MHz}, \mathrm{CDCl}_{3}\right) \delta_{\mathrm{H}}: 0.83\left(\mathrm{t}, J=6.9 \mathrm{~Hz}, 3 \mathrm{H}, \mathrm{CH}_{3}\right), 3.80-4.31(\mathrm{~m}, 2 \mathrm{H}$, 
$\left.\mathrm{CH}_{2}\right), 7.16-7.59$ (m, 14H, $\mathrm{OH}$ and aromatic). ${ }^{13} \mathrm{C}-\mathrm{NMR}\left(75 \mathrm{MHz}, \mathrm{CDCl}_{3}\right)$ $\delta_{\mathrm{C}}: 12.9,62.5,110.0,121.9,122.3,126.5,126.9,127.2,127.9,128.3,128.9$, $129.3,129.7,130.2,131.9,132.1,132.5,134.8,135.3,141.1,149.9,159.3$, 163.6, 169.4. IR (KBr) $v_{\max }: 732,1016,1081,1245,1480,1535,1590$, 1684, 2992, $3345 \mathrm{~cm}^{-1}$; Anal. Calcd for $\mathrm{C}_{26} \mathrm{H}_{19} \mathrm{Br}_{2} \mathrm{NO}_{3} \mathrm{~S}: \mathrm{C}, 53.35$; H, 3.27; $\mathrm{N}, 2.39$. Found: C, 53.30; H, 3.34; N, 2.31

Ethyl 2,6-Di(2,4-dichlorophenyl)-4-hydroxy-5-(phenylsulfanyl)-3pyridinecarboxylate $(\mathbf{5 g})$ : Isolated as solid $(0.080 \mathrm{~g}, 82 \%) ; \mathrm{mp} 83-84^{\circ} \mathrm{C}$; ${ }^{1} \mathrm{H}-\mathrm{NMR}\left(300 \mathrm{MHz}, \mathrm{CDCl}_{3}\right) \delta_{\mathrm{H}}: 0.93\left(\mathrm{t}, J=7.2 \mathrm{~Hz}, 3 \mathrm{H}, \mathrm{CH}_{3}\right), 3.99-4.14$ $\left(\mathrm{m}, 2 \mathrm{H}, \mathrm{CH}_{2}\right), 7.05-7.43\left(\mathrm{~m}, 12 \mathrm{H}, \mathrm{OH}\right.$ and aromatic). ${ }^{13} \mathrm{C}-\mathrm{NMR}(75 \mathrm{MHz}$, $\left.\mathrm{CDCl}_{3}\right) \delta_{\mathrm{C}}: 13.0,62.7,110.0,119.0,126.6,126.8,127.1,127.9,128.7$, 128.9, 129.3, 132.0, 133.1, 133.6, 134.2, 134.7, 134.9, 135.2, 136.5, 138.5, $157.5,163.2,169.5$. IR (KBr) $v_{\text {max }}: 736,1015,1084,1243,1478,1534$, 1594, 1680, 2988, $3343 \mathrm{~cm}^{-1}$; Anal. Calcd for $\mathrm{C}_{26} \mathrm{H}_{17} \mathrm{Cl}_{4} \mathrm{NO}_{3} \mathrm{~S}: \mathrm{C}, 55.24 ; \mathrm{H}$, 3.03; N, 2.48. Found: C, 55.32; H, 2.97; N, 2.55 .

Ethyl 4-Hydroxy-2,6-di(3-nitrophenyl)-5-(phenylsulfanyl)-3-pyridinecarboxylate $(\mathbf{5 h})$ : Isolated as solid $(0.074 \mathrm{~g}, 75 \%) ; \mathrm{mp} 75-76{ }^{\circ} \mathrm{C} ;{ }^{1} \mathrm{H}-\mathrm{NMR}$ $\left(300 \mathrm{MHz}, \mathrm{CDCl}_{3}\right) \delta_{\mathrm{H}}: 0.91\left(\mathrm{t}, J=7.2 \mathrm{~Hz}, 3 \mathrm{H}, \mathrm{CH}_{3}\right), 4.17(\mathrm{q}, J=7.2 \mathrm{~Hz}, 2 \mathrm{H}$, $\left.\mathrm{CH}_{2}\right), 7.07-7.66(\mathrm{~m}, 5 \mathrm{H}, \mathrm{OH}$ and aromatic), $7.85-8.48(\mathrm{~m}, 9 \mathrm{H}$, aromatic). ${ }^{13} \mathrm{C}-\mathrm{NMR}\left(75 \mathrm{MHz}, \mathrm{CDCl}_{3}\right) \delta_{\mathrm{C}}: 13.2,62.7,109.0,118.0,123.5,123.8$, $123.9,124.7,126.7,128.1,128.3,128.8,129.0,129.2,134.6,134.8,135.4$, $140.2,142.3,147.8,159.0,164.2,169.2$. IR (KBr) $v_{\text {max }}: 735,1016,1085$, 1246, 1360, 1481, 1535, 1590, 1683, 2991, 3346 cm-1 Anal. Calcd for $\mathrm{C}_{26} \mathrm{H}_{19} \mathrm{~N}_{3} \mathrm{O}_{7} \mathrm{~S}: \mathrm{C}, 60.34 ; \mathrm{H}, 3.70 ; \mathrm{N}, 8.12$. Found: $\mathrm{C}, 60.26 ; \mathrm{H}, 3.76 ; \mathrm{N}, 8.05$.

Ethyl 5-[(4-Chlorophenyl)sulfanyl]-4-hydroxy-2,6-diphenyl-3-pyridinecarboxylate (5i): Isolated as colorless crystals; $(0.075 \mathrm{~g}, 76 \%) ; \mathrm{mp} 92-93^{\circ} \mathrm{C}$; ${ }^{1} \mathrm{H}-\mathrm{NMR}\left(300 \mathrm{MHz}, \mathrm{CDCl}_{3}\right) \delta_{\mathrm{H}}: 0.79\left(\mathrm{t}, J=7.2 \mathrm{~Hz}, 3 \mathrm{H}, \mathrm{CH}_{3}\right), 3.99(\mathrm{q}, J=$ $\left.7.2 \mathrm{~Hz}, 2 \mathrm{H}, \mathrm{CH}_{2}\right), 6.58$ (br s, $\left.1 \mathrm{H}, \mathrm{OH}\right), 6.96-7.58$ (m, $14 \mathrm{H}$, aromatic). ${ }^{13} \mathrm{C}-$ NMR $\left(75 \mathrm{MHz}, \mathrm{CDCl}_{3}\right) \delta_{\mathrm{C}}: 13.5,61.5,108.4,108.5,127.9,128.1,128.4$, $128.9,129.0,129.1,129.5,129.8,132.2,134.3,142.0,156.4,160.4,165.2$, 169.5. IR (KBr) $v_{\text {max }}: 735,1025,1095,1248,1486,1541,1589,1689,2978$, $3351 \mathrm{~cm}^{-1}$; Anal. Calcd for $\mathrm{C}_{26} \mathrm{H}_{20} \mathrm{ClNO}_{3} \mathrm{~S}$ : C, 67.60; H, 4.36; N, 3.03 . Found: C, 67.68; $\mathrm{H}, 4.29 ; \mathrm{N}, 2.97$.

Ethyl 2,6-Di(4-chlorophenyl)-5-[(4-chlorophenyl)sulfanyl]-4-hydroxy-3pyridine-carboxylate $(\mathbf{5 j})$ : Isolated as colorless crystals; $(0.086 \mathrm{~g}, 87 \%) ; \mathrm{mp}$ $125-126^{\circ} \mathrm{C} ;{ }^{1} \mathrm{H}-\mathrm{NMR}\left(300 \mathrm{MHz}, \mathrm{CDCl}_{3}\right) \delta_{\mathrm{H}}: 0.88\left(\mathrm{t}, J=7.2 \mathrm{~Hz}, 3 \mathrm{H}, \mathrm{CH}_{3}\right)$, 4.03 (q, $\left.J=7.2 \mathrm{~Hz}, 2 \mathrm{H}, \mathrm{CH}_{2}\right), 6.65$ (br s, $\left.1 \mathrm{H}, \mathrm{OH}\right), 6.94-7.50(\mathrm{~m}, 12 \mathrm{H}$, aromatic). ${ }^{13} \mathrm{C}-\mathrm{NMR}\left(75 \mathrm{MHz}, \mathrm{CDCl}_{3}\right) \delta_{\mathrm{C}}: 13.7,61.7,108.2,108.7,127.8$, $128.3,128.6,129.9,130.3,130.9,132.5,133.8,135.2,135.3,138.7,140.4$, $156.7,160.5,164.0,169.1$. IR (KBr) $v_{\max }: 730,1029,1092,1243,1484$, 1532, 1594, 1696, 2995, $3347 \mathrm{~cm}^{-1}$; Anal. Calcd for $\mathrm{C}_{26} \mathrm{H}_{18} \mathrm{Cl}_{3} \mathrm{NO}_{3} \mathrm{~S}: \mathrm{C}$, 58.83; H, 3.42; N, 2.64. Found: C, 58.89; H, 3.46; N, 2.69.

Ethyl 5-[(4-Chlorophenyl)sulfanyl]-2,6-di(4-fluorophenyl)-4-hydroxy-3pyridine-carboxylate (5k): Isolated as colorless crystals; $(0.077 \mathrm{~g}, 78 \%) ; \mathrm{mp}$ $109-110^{\circ} \mathrm{C} ;{ }^{1} \mathrm{H}-\mathrm{NMR}\left(300 \mathrm{MHz}, \mathrm{CDCl}_{3}\right) \delta_{\mathrm{H}}: 0.88\left(\mathrm{t}, J=6.9 \mathrm{~Hz}, 3 \mathrm{H}, \mathrm{CH}_{3}\right)$, $4.03\left(\mathrm{q}, J=6.9 \mathrm{~Hz}, 2 \mathrm{H}, \mathrm{CH}_{2}\right), 6.61(\mathrm{brs}, 1 \mathrm{H}, \mathrm{OH}), 6.95-7.57(\mathrm{~m}, 12 \mathrm{H}$, aromatic). ${ }^{13} \mathrm{C}-\mathrm{NMR}\left(75 \mathrm{MHz}, \mathrm{CDCl}_{3}\right) \delta_{\mathrm{C}}: 13.7,61.6,108.2,108.4,114.9$, $115.2,115.5,127.8,128.7,129.9,130.7,130.8,131.4,131.6,132.4,134.0$, $136.5,138.2,156.6,160.5,162.0,164.1,165.1,166.3,169.3$. IR (KBr) $v_{\max }:$ 742, 1038, 1085, 1254, 1491, 1540, 1598, 1690, 2991, $3340 \mathrm{~cm}^{-1}$; Anal. Calcd for $\mathrm{C}_{26} \mathrm{H}_{18} \mathrm{ClF}_{2} \mathrm{NO}_{3} \mathrm{~S}: \mathrm{C}, 62.71 ; \mathrm{H}, 3.64 ; \mathrm{N}, 2.81$. Found: $\mathrm{C}, 62.65 ; \mathrm{H}$ $3.60 ; \mathrm{N}, 2.87$.

Ethyl 5-[(4-Chlorophenyl)sulfanyl]-4-hydroxy-2,6-di(4-methylphenyl)-3pyridine-carboxylate (5I): Isolated as colorless crystals; $(0.071 \mathrm{~g}, 72 \%)$; $\mathrm{mp}$ $105-106{ }^{\circ} \mathrm{C} ;{ }^{1} \mathrm{H}-\mathrm{NMR}\left(300 \mathrm{MHz}, \mathrm{CDCl}_{3}\right) \delta_{\mathrm{H}}: 0.78\left(\mathrm{t}, J=6.9 \mathrm{~Hz}, 3 \mathrm{H}, \mathrm{CH}_{3}\right)$, $2.31\left(\mathrm{~s}, 1 \mathrm{H}, \mathrm{CH}_{3}\right), 2.35\left(\mathrm{~s}, 1 \mathrm{H}, \mathrm{CH}_{3}\right), 3.99\left(\mathrm{q}, J=6.9 \mathrm{~Hz}, 2 \mathrm{H}, \mathrm{CH}_{2}\right), 6.87-$ $7.61\left(\mathrm{~m}, 12 \mathrm{H}\right.$, aromatic). ${ }^{13} \mathrm{C}-\mathrm{NMR}\left(75 \mathrm{MHz}, \mathrm{CDCl}_{3}\right) \delta_{\mathrm{C}}: 13.5,21.7,21.8$, $62.0,108.6,112.2,126.6,127.2,128.1,128.9,129.0,129.1,129.4130 .0$, $132.7,133.2,140.0,157.3,160.4,165.2,168.5$. IR (KBr) $v_{\max }: 731,1046$, 1081, 1250, 1487, 1549, 1592, 1696, 2995, $3350 \mathrm{~cm}^{-1}$; Anal. Calcd for $\mathrm{C}_{28} \mathrm{H}_{24} \mathrm{ClNO}_{3} \mathrm{~S}$ : C, 68.63; H, 4.94; N, 2.86. Found: $\mathrm{C}, 68.59 ; \mathrm{H}, 4.88 ; \mathrm{N}$, 2.90 .

Ethyl 2,6-Di(2-chlorophenyl)-5-[(4-chlorophenyl)sulfanyl]-4-hydroxy-3pyridine-carboxylate $(\mathbf{5 m})$ : Isolated as colorless crystals; $(0.079 \mathrm{~g}, 80 \%) ; \mathrm{mp}$ $112-113{ }^{\circ} \mathrm{C} ;{ }^{1} \mathrm{H}-\mathrm{NMR}\left(300 \mathrm{MHz}, \mathrm{CDCl}_{3}\right) \delta_{\mathrm{H}}: 0.85\left(\mathrm{t}, J=7.2 \mathrm{~Hz}, 3 \mathrm{H}, \mathrm{CH}_{3}\right)$, $3.94-4.25\left(\mathrm{~m}, 2 \mathrm{H}, \mathrm{CH}_{2}\right), 6.95-7.49\left(\mathrm{~m}, 12 \mathrm{H}\right.$, aromatic). ${ }^{13} \mathrm{C}-\mathrm{NMR}(75$ $\left.\mathrm{MHz}, \mathrm{CDCl}_{3}\right) \delta_{\mathrm{C}}: 13.6,61.7,108.7,112.8,126.9,127.4,127.6,128.9$, $129.2,129.5,129.7,129.9,130.1,130.6,132.2,132.7,133.2,134.5,139.7$, $141.7,156.2,159.2,163.8,168.5$. IR (KBr) $v_{\max }: 738,1041,1097,1263$, 1495, 1542, 1581, 1690, 2998, $3353 \mathrm{~cm}^{-1}$; Anal. Calcd for $\mathrm{C}_{26} \mathrm{H}_{18} \mathrm{Cl}_{3} \mathrm{NO}_{3} \mathrm{~S}$ : C, $58.83 ; \mathrm{H}, 3.42 ; \mathrm{N}, 2.64$. Found: C, $58.88 ; \mathrm{H}, 3.37 ; \mathrm{N}, 2.59$.

Ethyl 2,6-Di(2-bromophenyl)-5-[(4-chlorophenyl)sulfanyl]-3-pyridinecarboxylate $(\mathbf{5 n})$ : Isolated as solid $(0.069 \mathrm{~g}, 70 \%) ; \mathrm{mp} 77-78^{\circ} \mathrm{C} ;{ }^{1} \mathrm{H}-\mathrm{NMR}$ $\left(300 \mathrm{MHz}, \mathrm{CDCl}_{3}\right) \delta_{\mathrm{H}}: 0.84\left(\mathrm{t}, J=7.2 \mathrm{~Hz}, 3 \mathrm{H}, \mathrm{CH}_{3}\right), 4.00-4.28(\mathrm{~m}, 2 \mathrm{H}$,
$\left.\mathrm{CH}_{2}\right), 6.98-7.61\left(\mathrm{~m}, 13 \mathrm{H}, \mathrm{OH}\right.$ and aromatic). ${ }^{13} \mathrm{C}-\mathrm{NMR}\left(75 \mathrm{MHz}, \mathrm{CDCl}_{3}\right)$ $\delta_{\mathrm{C}}: 12.9,62.5,110.0,121.9,122.4,126.5,126.9,127.2,127.9,128.3,128.9$, $129.3,129.7,130.2,131.9,132.1,132.5,134.8,135.3,141.1,143.1,149.9$, 159.4, 169.5. IR (KBr) $v_{\max }: 736,1043,1096,1265,1497,1540,1582$, 1688, 2997, $3351 \mathrm{~cm}^{-1}$; Anal. Calcd for $\mathrm{C}_{26} \mathrm{H}_{18} \mathrm{Br}_{2} \mathrm{ClNO}_{3} \mathrm{~S}$ : C, 50.39; $\mathrm{H}$, 2.93 ; N, 2.26. Found: C, 50.45; H, 2.99; N, 2.18 .

Ethyl 5-[(4-Chlorophenyl)sulfanyl]-2,6-di(2,4-dichlorophenyl)-3pyridinecarboxylate (5o): Isolated as solids $(0.078 \mathrm{~g}, 79 \%)$; mp $109-$ $110{ }^{\circ} \mathrm{C} ;{ }^{1} \mathrm{H}-\mathrm{NMR}\left(300 \mathrm{MHz}, \mathrm{CDCl}_{3}\right) \delta_{\mathrm{H}}: 0.93\left(\mathrm{t}, J=7.2 \mathrm{~Hz}, 3 \mathrm{H}, \mathrm{CH}_{3}\right)$, $4.09-4.26\left(\mathrm{~m}, 2 \mathrm{H}, \mathrm{CH}_{2}\right), 7.04-7.43(\mathrm{~m}, 11 \mathrm{H}, \mathrm{OH}$ and aromatic $) .{ }^{13} \mathrm{C}-$ NMR $\left(75 \mathrm{MHz}, \mathrm{CDCl}_{3}\right) \delta_{\mathrm{C}}: 13.0,62.8,110.0,118.5,126.9,127.1,128.3$, $128.6,129.0,129.4,130.2,130.5,132.6,133.0,133.2,133.6,134.8,135.3$, $136.5,138.5,157.0,163.3,168.8,169.5$. IR (KBr) $v_{\max }: 737,1044,1098$, 1260, 1496, 1545, 1579, 1691, 2995, $3356 \mathrm{~cm}^{-1}$; Anal. Calcd for $\mathrm{C}_{26} \mathrm{H}_{16} \mathrm{Cl}_{5} \mathrm{NO}_{3} \mathrm{~S}$ : C, 52.07; H, 2.69; N, 2.34. Found: $\mathrm{C}, 52.15 ; \mathrm{H}, 2.62 ; \mathrm{N}$, 2.41 .

Ethyl 4-Hydroxy-5-[(4-methylphenyl)sulfanyl]-2,6-diphenyl-3-pyridinecarboxylate (5p): Isolated as colorless crystals; $(0.069 \mathrm{~g}, 70 \%) ; \mathrm{mp} 95-96{ }^{\circ} \mathrm{C}$; ${ }^{1} \mathrm{H}-\mathrm{NMR}\left(300 \mathrm{MHz}, \mathrm{CDCl}_{3}\right) \delta_{\mathrm{H}}: 0.77\left(\mathrm{t}, J=6.9 \mathrm{~Hz}, 3 \mathrm{H}, \mathrm{CH}_{3}\right), 2.30(\mathrm{~s}, 1 \mathrm{H}$, $\left.\mathrm{CH}_{3}\right), 3.97$ (q, $\left.J=6.9 \mathrm{~Hz}, 2 \mathrm{H}, \mathrm{CH}_{2}\right), 6.95-7.54$ (m, $14 \mathrm{H}$, aromatic). ${ }^{13} \mathrm{C}-$ NMR $\left(75 \mathrm{MHz}, \mathrm{CDCl}_{3}\right) \delta_{\mathrm{C}}: 13.5,21.4,61.9,108.5,111.3,126.6,127.4$, $128.2,128.5,129.0,129.7,130.7,130.9,137.0,137.7,139.2,157.3,160.4$, 165.2, 168.5. IR (KBr) $v_{\text {max }}: 748,1056,1091,1260,1499,1553,1587$, 1681, 2987, $3357 \mathrm{~cm}^{-1}$; Anal. Calcd for $\mathrm{C}_{27} \mathrm{H}_{23} \mathrm{NO}_{3} \mathrm{~S}: \mathrm{C}, 73.44 ; \mathrm{H}, 5.25 ; \mathrm{N}$, 3.17. Found: $\mathrm{C}, 73.49 ; \mathrm{H}, 5.31 ; \mathrm{N}, 3.23$

Ethyl 2,6-Di(4-chlorophenyl)-4-hydroxy-5-[(4-methylphenyl)sulfanyl]-3pyridine-carboxylate $\mathbf{( 5 q})$ : Isolated as colorless crystals; $(0.078 \mathrm{~g}, 79 \%) ; \mathrm{mp}$ $119-120^{\circ} \mathrm{C} ;{ }^{1} \mathrm{H}-\mathrm{NMR}\left(300 \mathrm{MHz}, \mathrm{CDCl}_{3}\right) \delta_{\mathrm{H}}: 0.87\left(\mathrm{t}, J=7.2 \mathrm{~Hz}, 3 \mathrm{H}, \mathrm{CH}_{3}\right)$, $2.29\left(\mathrm{~s}, 1 \mathrm{H}, \mathrm{CH}_{3}\right), 4.01\left(\mathrm{q}, J=7.2 \mathrm{~Hz}, 2 \mathrm{H}, \mathrm{CH}_{2}\right), 6.66(\mathrm{br} \mathrm{s}, 1 \mathrm{H}, \mathrm{OH}), 6.93-$ 7.51 (m, $12 \mathrm{H}$, aromatic). ${ }^{13} \mathrm{C}-\mathrm{NMR}\left(75 \mathrm{MHz}, \mathrm{CDCl}_{3}\right) \delta_{\mathrm{C}}: 13.7,21.3,61.6$, $108.1,109.7,126.8,128.3,128.5,130.3,130.6,131.1,131.6,135.0,135.2$, $136.5,138.9,140.6,156.7,160.2,163.8,169.2$. IR (KBr) $v_{\max }: 742,1051$, 1084, 1267, 1506, 1559,1582, 1678, 2993, $3349 \mathrm{~cm}^{-1}$; Anal. Calcd for $\mathrm{C}_{27} \mathrm{H}_{21} \mathrm{Cl}_{2} \mathrm{NO}_{3} \mathrm{~S}: \mathrm{C}, 63.53 ; \mathrm{H}, 4.15 ; \mathrm{N}, 2.74$. Found: $\mathrm{C}, 63.59 ; \mathrm{H}, 4.11 ; \mathrm{N}$, 2.68 .

Ethyl 2,6-Di(4-fluorophenyl)-4-hydroxy-5-[(4-methylphenyl)sulfanyl]-3pyridine-carboxylate (5r): Isolated as colorless crystals; $(0.071 \mathrm{~g}, 72 \%) ; \mathrm{mp}$ $107-108^{\circ} \mathrm{C}$; ${ }^{1} \mathrm{H}-\mathrm{NMR}\left(300 \mathrm{MHz}, \mathrm{CDCl}_{3}\right) \delta_{\mathrm{H}}: 0.87\left(\mathrm{t}, J=7.2 \mathrm{~Hz}, 3 \mathrm{H}, \mathrm{CH}_{3}\right)$, 2.29 (s, $\left.1 \mathrm{H}, \mathrm{CH}_{3}\right), 4.01$ (q, J=7.2 Hz, 2H, $\mathrm{CH}_{2}$ ), 6.63 (br s, $\left.1 \mathrm{H}, \mathrm{OH}\right), 6.93-$ 7.57 (m, $12 \mathrm{H}$, aromatic). ${ }^{13} \mathrm{C}-\mathrm{NMR}\left(75 \mathrm{MHz}, \mathrm{CDCl}_{3}\right) \delta_{\mathrm{C}}: 13.7,21.3,61.5$, $108.1,109.4,114.8,115.1,115.5,115.5,126.8,130.5,130.7,130.8,131.5$, 131.6, 131.7, 136.5, 136.6, 138.2, 156.6, 160.2, 161.9, 164.9, 169.3. IR (KBr) $V_{\max }: 740,1056,1079,1260,1502,1568,1589,1681,2988,3341 \mathrm{~cm}^{-1}$; Anal. Calcd for $\mathrm{C}_{27} \mathrm{H}_{21} \mathrm{~F}_{2} \mathrm{NO}_{3} \mathrm{~S}$ : C, 67.91; H, 4.43; N, 2.93. Found: C, 67.98; $\mathrm{H}, 4.49 ; \mathrm{N}, 2.89$.

Ethyl 4-Hydroxy-2,6-di(4-methylphenyl)-5-[(4-methylphenyl)sulfanyl]-3pyridine-carboxylate $(\mathbf{5 s})$ : Isolated as viscous liquid; $(0.069 \mathrm{~g}, 70 \%) ;{ }^{1} \mathrm{H}-$ NMR $\left(300 \mathrm{MHz}, \mathrm{CDCl}_{3}\right) \delta_{\mathrm{H}}: 0.80\left(\mathrm{t}, J=6.6 \mathrm{~Hz}, 3 \mathrm{H}, \mathrm{CH}_{3}\right), 2.26(\mathrm{~s}, 1 \mathrm{H}$, $\left.\mathrm{CH}_{3}\right), 2.30\left(\mathrm{~s}, 1 \mathrm{H}, \mathrm{CH}_{3}\right), 2.35\left(\mathrm{~s}, 1 \mathrm{H}, \mathrm{CH}_{3}\right), 3.97\left(\mathrm{q}, J=6.6 \mathrm{~Hz}, 2 \mathrm{H}, \mathrm{CH}_{2}\right)$, $6.54(\mathrm{brs}, 1 \mathrm{H}, \mathrm{OH}), 6.94-7.46\left(\mathrm{~m}, 12 \mathrm{H}\right.$, aromatic). ${ }^{13} \mathrm{C}-\mathrm{NMR}(75 \mathrm{MHz}$, $\left.\mathrm{CDCl}_{3}\right) \delta_{\mathrm{c}}: 13.7,21.4,21.7,21.8,61.5,108.2,109.0,126.7,128.7,128.9$, $129.0,129.6,130.5,132.2,136.2,137.6,138.8,138.9,139.0,156.5,161.1$, 164.9, 169.7. IR $\left(\mathrm{CHCl}_{3}\right) v_{\text {max }}: 745,1052,1082,1268,1513,1561,1582$, 1676, 2980, $3357 \mathrm{~cm}^{-1}$; Anal. Calcd for $\mathrm{C}_{29} \mathrm{H}_{27} \mathrm{NO}_{3} \mathrm{~S}: \mathrm{C}, 74.17 ; \mathrm{H}, 5.80 ; \mathrm{N}$, 2.98. Found: C, 74.23; H, 5.86; N, 2.91

Ethyl 2,6-Di(2-chlorophenyl)-4-hydroxy-5-[(4-methylphenyl)sulfanyl]-3pyridine-carboxylate $(\mathbf{5 t})$ : Isolated as colorless crystals; $(0.073 \mathrm{~g}, 74 \%) ; \mathrm{mp}$ 98-99 ${ }^{\circ} \mathrm{C} ;{ }^{1} \mathrm{H}-\mathrm{NMR}\left(300 \mathrm{MHz}, \mathrm{CDCl}_{3}\right) \delta_{\mathrm{H}}: 0.84\left(\mathrm{t}, J=7.2 \mathrm{~Hz}, 3 \mathrm{H}, \mathrm{CH}_{3}\right)$, $2.28\left(\mathrm{~s}, 1 \mathrm{H}, \mathrm{CH}_{3}\right), 3.91-4.20\left(\mathrm{~m}, 2 \mathrm{H}, \mathrm{CH}_{2}\right), 6.77-7.80(\mathrm{~m}, 12 \mathrm{H}$, aromatic). ${ }^{13} \mathrm{C}-\mathrm{NMR}\left(75 \mathrm{MHz}, \mathrm{CDCl}_{3}\right) \delta_{\mathrm{C}}: 13.5,21.3,61.8,108.5,112.6$, $126.7,127.2,127.4,128.7,129.0,129.3,129.5,129.7,130.0,130.4,132.0$ $132.5,133.0,134.3,139.6,141.6,156.3,159.0,163.6,169.4$. IR (KBr) $v_{\max }$ : 749, 1058, 1076, 1271, 1507, 1565, 1589, 1681, 2973, $3348 \mathrm{~cm}^{-1}$; Anal. Calcd for $\mathrm{C}_{27} \mathrm{H}_{21} \mathrm{Cl}_{2} \mathrm{NO}_{3} \mathrm{~S}$ : C, 63.53; H, 4.15; N, 2.74. Found: C, 63.48; H, $4.21 ; \mathrm{N}, 2.80$.

Ethyl 2,6-Di(2,4-dichlorophenyl)-4-hydroxy-5-[(4-methylphenyl)-3pyridinecarboxylate $(\mathbf{5 u})$ : Isolated as solid $(0.080 \mathrm{~g}, 81 \%)$; mp $76-77^{\circ} \mathrm{C}$; ${ }^{1} \mathrm{H}-\mathrm{NMR}\left(300 \mathrm{MHz}, \mathrm{CDCl}_{3}\right) \delta_{\mathrm{H}}: 0.92\left(\mathrm{t}, J=7.2 \mathrm{~Hz}, 3 \mathrm{H}, \mathrm{CH}_{3}\right), 2.23(\mathrm{~s}, 3 \mathrm{H}$, $\left.\mathrm{CH}_{3}\right), 3.94-4.14\left(\mathrm{~m}, 2 \mathrm{H}, \mathrm{CH}_{2}\right), 7.03-7.49\left(\mathrm{~m}, 11 \mathrm{H}, \mathrm{OH}\right.$, aromatic). ${ }^{13} \mathrm{C}-$ NMR $\left(75 \mathrm{MHz}, \mathrm{CDCl}_{3}\right) \delta_{\mathrm{C}}: 13.0,21.0,62.6,110.0,119.2,126.8,127.0$, $128.3,128.6,129.3,129.4,129.7,130.0,130.7,131.0,132.5,133.1,134.8$ $135.1,136.7,138.7,157.0,163.1,168.8,169.5$. IR (KBr) $v_{\max }: 745,1057$, 1078, 1270, 1508, 1562,1590, 1685, 2976, $3346 \mathrm{~cm}^{-1}$; Anal. Calcd for $\mathrm{C}_{27} \mathrm{H}_{19} \mathrm{Cl}_{4} \mathrm{NO}_{3} \mathrm{~S}$ : C, 55.98; H, 3.31; N, 2.42. Found: C, 55.92; H, 3.25; N, 
2.48 .

Ethyl 4-Hydroxy-5-[(4-methylphenyl)sulfanyl]-2,6-di(3-nitrophenyl)-3pyridine-carboxylate $(\mathbf{5 v})$ : Isolated as solid $(0.077 \mathrm{~g}, 78 \%) ; \mathrm{mp} 120-121$ ${ }^{\circ} \mathrm{C}$; ${ }^{1} \mathrm{H}-\mathrm{NMR}\left(300 \mathrm{MHz}, \mathrm{CDCl}_{3}\right) \delta_{\mathrm{H}}: 0.91\left(\mathrm{t}, J=7.2 \mathrm{~Hz}, 3 \mathrm{H}, \mathrm{CH}_{3}\right), 2.28$ (s, $\left.3 \mathrm{H}, \mathrm{CH}_{3}\right), 4.17\left(\mathrm{q}, J=7.2 \mathrm{~Hz}, 2 \mathrm{H}, \mathrm{CH}_{2}\right), 6.91-8.52(\mathrm{~m}, 13 \mathrm{H}, \mathrm{OH}$, aromatic). ${ }^{13} \mathrm{C}-\mathrm{NMR}\left(75 \mathrm{MHz}, \mathrm{CDCl}_{3}\right) \delta_{\mathrm{C}}: 13.2,20.9,62.6,109.3,117.5$, 123.8, 124.7, 127.3, 128.3, 128.7, 129.0, 129.9, 130.3, 130.5, 131.1, 134.6, $135.4,136.9,140.3,142.2,147.7,158.5,162.5,168.9,169.3 . \mathrm{IR}(\mathrm{KBr}) v_{\max }$ : 733, 1019, 1084, 1245, 1357, 1483, 1539, 1592, 1688, 2994, $3347 \mathrm{~cm}^{-1}$; Anal. Calcd for $\mathrm{C}_{27} \mathrm{H}_{21} \mathrm{~N}_{3} \mathrm{O}_{7} \mathrm{~S}$ : C, 61.01; H, 3.98; N, 7.91. Found: C, 61.07; H, 3.95; N, 7.98 .

In-Vitro Antimycobacterial Activity All compounds were screened for their in vitro anti-mycobacterial activity against MTB, in Middlebrook 7H1 lagar medium supplemented with OADC by agar dilution method similar to that recommended by the National Committee for Clinical Laboratory Standards for the determination of MIC in triplicate. ${ }^{51)}$ The MIC is defined as the minimum concentration of compound required to give complete inhibition of bacterial growth.

Acknowledgements S.P. thanks the Department of Science and Technology, New Delhi, for funding for a major research project (No. SR/S1/OC70/2006) and for funds under (i) IRHPA program for funds for the purchase of a high resolution NMR spectrometer and (ii) FIST program and the University Grants Commission, New Delhi, for (i) funds under the DRS and ASIST programs and (ii) for funding for a major research project [F. No. 36155/2008(SR)]. RSK thanks CSIR, New Delhi for Senior Research Fellowship and SMR thanks UGC, New Delhi for Junior Research Fellowship.

\section{References and Notes}

1) Tawara J. N., Lorenz P., Stermitz F. R., J. Nat. Prod., 62, 321-323 (1999).

2) Watson P. S., Jiang B., Scott B., Org. Lett., 2, 3679-3681 (2000).

3) Brooks C. A., Comins D. L., Tetrahedron Lett., 41, 3551-3553 (2000).

4) Mukhtar T. A., Wright G. D., Chem. Rev., 105, 529-542 (2005).

5) Richardo G. J., Juan B. C., Mario R. A., Rolder M., Peinado C. R., Fernando. Spen., 47, 168-172 (1979).

6) Jerom B. R., Spencer K. H., Eur. Pat. Appl. EP 277794 (1998).

7) Perumal R. V., Adiraj M., Shanmugapandian P., Indian Drugs, 38, $156-159$ (2001).

8) Ganellin C. R., Spickett, R. G., J. Med. Chem., 8, 619-625 (1965).

9) Hangenbach R. E., Gysin H., Experentia, 8, 184-185 (1952).

10) Heana B., Dobre V., Niculesar-Duvaz I., J. Prakt. Chem., 327, 667674 (1985).

11) Mobio I. G., Soldatenkov A. T., Federov V. O., Ageer E. A., Sergeeva N. D., Lin S., Stashenko E. E., Prostakov N. S., Andreeva E. I., Khim. Farm. Zh., 23, 421-427 (1989).

12) Angle S. R., Breitenbucher J. G., "Natural Products Chemistry: Stereoselective Synthesis. Atta-ur-Rahman," 16th ed., Part I, Elsevier, New York, 1995, pp. 453-502.

13) Grishina G. V., Gaidorova E. L., Zefirov N. S., Chem. Heterocycl. Compd., 30, 1401-1426 (1994).

14) Wang C. L., Wuorola M. A., Org. Prep. Proceed. Int., 24, 585-621 (1992).

15) Fleet G. W. J., Ramsden N. G., Witty D. R., Tetrahedron, 45, 319-326 (1990).

16) Winkler D. A., Holan G., J. Med. Chem., 32, 2084-2089 (1989).

17) Fleet G. W. J., Ramsden N. G., Dwek R. A., Rademacher T. W., Nash R. J., Green D. S. C., Winchester B., J. Chem. Soc. Chem. Commun., 7, 483-485 (1988).

18) Beeler A. B., Gadepalli R. S. V. S., Steyn S., Castagnoli N., Rimoldi J. M., Bioorg. Med. Chem., 11, 5229-5234 (2003).

19) Deskus J. A., Epperson J. R., Charles P. S., Joseph A. C., Dextraze P., Qian-Cutrone J., Gao Q., Ma B., Beno B. R., Mattson G. K., Molski T. F., Krause R. G., Taber M. T., Lodge N. J., Mattson R. J., Bioorg. Med. Chem. Lett., 17, 3099-3104 (2007).

20) Gwaltney S. L., O’Connor S. J., Nelson L. T. J., Sullivan G. M., Imade H., Wang W., Hasvold L., Li Q., Cohen J., Gu W.-Z., Tahir S. K., Bauch J., Marsh K., Ng S.-C., Frost D. J., Zhang H., Muchmore S., Jakob C. G., Stoll V., Hutchins C., Bioorg. Med. Chem. Lett., 13, 1359-1362 (2003).

21) Kamei K., Maeda N., Katsuragi-Ogino R., Koyama M., Nakajima M.,
Tatsuoka T., Ohno T., Inoue T., Bioorg. Med. Chem. Lett., 15, 29902993 (2005).

22) Hisaki M., Kashima K., Sakamoto Y., Hojo M., Katayama O., Hata H., Eur. Pat. Appl. EP 220653 (1987).

23) Attia A., Michael M., Pharmazie, 37, 551-553 (1982)

24) Quintela J. M., Peinador C., Botana L., Estevez M., Riguera R., Bioorg. Med. Chem., 5, 1543-1553 (1997).

25) Zhu G. D., Arendsen D. L., Gunawardana I. W., Boyd S. A., Stewart A. O., Fry D. G., Cool B. L., Kifle L., Schaefer V., Meuth J., Marsh K. C., Kempf-Grote A. J., Kilgannon P., Gallatin W. M., Okasinski G. F., J. Med. Chem., 44, 3469-3487 (2001).

26) Roth H. J., Kleeman A., "Pharmaceutical Chemistry," John Wiley \& Sons, New York, 1988.

27) Bashford K. E., Burton M. B., Cameron S., Cooper A. L., Hogg R. D., Kane P. D., MacManus D. A., Matrunola C. A., Moody C. J., Robertson A. A. B., Warne M. R., Tetrahedron Lett., 44, 1627-1629 (2003).

28) Raw S. A., Taylor R. J. K., Chem. Commun., 2004, 508-509 (2004).

29) Constable E. C., Housecroft C. E., Neuburger M., Phillips D., Raithby P. R., Schofield E., Sparr E., Tocher D. A., Zehnder M., Zimmermann Y., J. Chem. Soc. Dalton Trans., 2000, 2219-2228 (2000).

30) Cave G. W. V., Hardie M. J., Roberts B. A., Raston C. L., Eur. J. Org. Chem., 2001, 3227-3231 (2001).

31) Cutshall N. S., Kucera K. A., Ursion R., Latham J., Ihle N. C., Bioorg. Med. Chem. Lett., 12, 1517-1520 (2002).

32) Comins D. L., Smith E. D., Tetrahedron Lett., 47, 1449-1451 (2006).

33) http://www.who.int/mediacentre/news/releases/2009/tuberculosis_report_20090324/en/index.html.

34) Janin Y. L., Bioorg. Med. Chem., 15, 2479-2513 (2007).

35) Gutierrez-Lugo M. T., Bewley C. A., J. Med. Chem., 51, 2606-2612 (2008).

36) O’Brien R. J., Nunn P. P., Am. J. Respir. Crit. Care Med., 163, 10551058 (2001).

37) Ranjith Kumar R., Perumal S., Senthilkumar P., Yogeeswari P., Sriram D., J. Med. Chem., 51, 5731-5735 (2008).

38) Ranjith Kumar R., Perumal S., Senthilkumar P., Yogeeswari P., Sriram D., Tetrahedron, 64, 2962-2971 (2008).

39) Ranjith Kumar R., Perumal S., Senthilkumar P., Yogeeswari P., Sriram D., Bioorg. Med. Chem. Lett., 17, 6459-6462 (2007).

40) Karthikeyan S. V., Perumal S., Krithika A. S., Yogeeswari P., Sriram D., Bioorg. Med. Chem. Lett., 19, 3006-3009 (2009).

41) Ranjith Kumar R., Perumal S., Manju S. C., Bhatt P., Yogeeswari P., Sriram D., Bioorg. Med. Chem. Lett., 19, 3461-3465 (2009).

42) Ranjith Kumar R., Perumal S., Senthilkumar P., Yogeeswari P., Sriram D., Eur. J. Med. Chem., 44, 3821-3829 (2009).

43) Balamurugan K., Perumal S., Reddy, A. S. K., Yogeeswari P., Sriram D., Tetrahedron Lett., 50, 6191-6195 (2009).

44) Noller C. R., Baliah V., J. Am. Chem. Soc., 70, 3853-3855 (1948),

45) Shimada K., Kaburagi Y., Fukuyama T., J. Am. Chem. Soc., 125, $4048-4049$ (2003).

46) Suresh J., Suresh Kumar R., Perumal S., Natarajan S., Acta Cryst., E63, o777—o 779 (2007).

47) Suresh J., Suresh Kumar R., Perumal S., Natarajan S., Acta Cryst., E63, o1377-01379 (2007).

48) Suresh J., Suresh Kumar R., Perumal S., Natarajan S., Acta Cryst., E63, o2140-02141 (2007).

49) Suresh J., Suresh Kumar R., Perumal S., Natarajan S., Acta Cryst., E63, o1375-01376 (2007).

50) Suresh J., Suresh Kumar R., Perumal S., Mostad A., Natarajan S., Acta Cryst., C63, o141—o144 (2007).

51) Ten fold serial dilutions of each test compound/drug were incorporated into Middlebrook 7H11 agar medium with OADC Growth Supplement. Inoculum of M. tuberculosis $\mathrm{H}_{37} \mathrm{Rv}$ were prepared from fresh Middlebrook 7H11 agar slants with OADC Growth Supplement adjusted to $1 \mathrm{mg} / \mathrm{ml}$ (wet weight) in Tween $80(0.05 \%)$ saline diluted to $10^{-2}$ to give a concentration of approximately $10^{7} \mathrm{cfu} / \mathrm{ml}$. A $5 \mu \mathrm{l}$ amount of bacterial suspension was spotted into $7 \mathrm{H} 11$ agar tubes containing 10-fold serial dilutions of drugs per milliliter. The tubes were incubated at $37^{\circ} \mathrm{C}$, and final readings were recorded after $28 \mathrm{~d}$. The minimum inhibitory concentration (MIC) is defined as the minimum concentration of compound required to give complete inhibition of bacterial growth. 